\title{
Classification of spin structures on the noncommutative $n$-torus*
}

\author{
Jan Jitse Venselaar
}

\begin{abstract}
We classify spin structures on the noncommutative torus and find that the noncommutative $n$-torus has $2^{n}$ spin structures corresponding to isospectral deformations of spin structures on the commutative $n$-torus. For $n \geq 4$ the classification depends on Connes' spin manifold theorem. In addition, we study unitary equivalences of these spin structures.
\end{abstract}

Mathematics Subject Classification (2010). 58B34; 46L87.

Keywords. Spectral geometry, noncommutative geometry, real spectral triples.

\section{Introduction}

The different possible spin structures on a differentiable manifold were classified in the work of Milnor [27]; for example, on a (commutative) $n$-torus there exist $2^{n}$ inequivalent spin structures. No such general classification of spin structures is currently known in noncommutative geometry - this amounts to classifying the possible real spectral triple structures on a $\mathrm{C}^{*}$-algebra. In this paper we prove that there exist precisely $2^{n}$ different real spectral triples on a noncommutative $n$-torus, and that these structures are isospectral deformations of spin structures on the commutative $n$-torus.

The noncommutative torus $A\left(\mathbb{T}_{\theta}^{n}\right)$, or irrational rotation algebra, is one of the first nontrivial examples of a noncommutative topological manifold, given as a deformation of the usual commutative torus [17], [31], [16]. The parameter $\theta$ is a number for a noncommutative 2-torus, and an antisymmetric $(n \times n)$-matrix for higher-dimensional tori.

The analog of putting a spin structure and a metric on this algebra is to enhance it into a real spectral triple in the sense of Connes [8], [9]. This introduces a set of extra parameters $\boldsymbol{\tau}^{i}$, which are the analogue of the size of the torus. The noncommutative $n$-torus, both topologically and with spin structure, has found many applications in mathematical physics, for example [4], [25] and [3]. A noncommutative spin structure can certainly be constructed by deforming a spin structure on the commutative $n$ torus [13], so the question becomes whether this deformation gives all possible spin structures on the noncommutative $n$-torus.

\footnotetext{
*The author thanks Gunther Cornelissen for discussions and encouragement, and the referee for pertinent remarks.
} 
In dimension 2, the problem was solved by Paschke and Sitarz [28], Theorem 2.5, who showed that a noncommutative 2-torus admits exactly 4 different real spectral triples (which are deformations of spin structures on the commutative torus). This result can be reformulated as follows: any real spectral triple which is equivariant with respect to a 2-torus action in the sense of [34] (see Section 2.1), is an isospectral deformation of a spin structure on a commutative 2-torus. Note that an equivariant action of $n$-torus is different from an $n$-torus action as in [11], the former is a condition on the spectral triple, the latter is an action along which the algebra is deformed.

Our first result is that the theorem of Paschke and Sitarz holds true in arbitrary dimension:

Theorem A. All irreducible real spectral triples with an equivariant $n$-torus actions are isospectral deformations of spin structures on an n-torus.

The proof of [28], Theorem 2.5, does not generalize readily to higher dimensions. Rather than working with the grading operator, which only gives nontrivial conditions in the even-dimensional case, we use the reality operator first. Then we establish that out of several possible candidate structures only one satisfies the growth condition and compact resolvent condition on the Dirac operator. Also, our proof uses at a crucial point Connes' reconstruction theorem [10], Theorem 11.5. We describe the spin structures explicitly in Theorem $\mathrm{C}$.

In a celebrated paper [1] (see also [2], Theorem 1), Adams used the classification of independent vector fields on spheres to deduce elementary results on Radon-Hurwitz numbers of certain classes of matrices. Similarly, our Theorem A can be used to prove the following elementary result on Hermitian matrices, for which we do not know an elementary proof:

Corollary 1. A set of $\left(2^{b} \times 2^{b}\right)$ Hermitian matrices $\left\{A_{i}\right\}_{i=1}^{n}$, where $n=2 b+1$, such that the equation

$$
\operatorname{det}\left(\sum_{i} x_{i} A_{i}\right)=0
$$

only has the zero solution $\left(x_{i}=0\right)_{i=1}^{n}$ in $\mathbb{R}^{n}$ generate a Clifford algebra if and only if

$$
\sum_{\sigma \in S_{n}} \operatorname{sign}(\sigma) \prod_{i}^{n} A_{\sigma(i)}=\lambda \operatorname{Id}_{k},
$$

for some nonzero $\lambda \in \mathbb{R}$.

Once we have obtained a classification, we study equivalences between different real spectral triples on the same noncommutative $n$-torus. For a commutative $n$-torus, the diffeomorphisms of a torus act affine on the set of spin structures identified with the vector space $\mathbb{Z}_{2}^{n}$, as shown in [15]. In particular, for the commutative 2-torus there are two orbits, one consisting of one element, and the other consisting of three elements. In the case of the noncommutative torus the full diffeomorphism group is 
not known when $n>2$. Restricting to inner automorphisms of the algebra, we can show the following.

Theorem B. Except for a set of $\theta$ of measure 0, the different spin structures of the smooth noncommutative $n$-torus $\mathcal{A}\left(\mathbb{T}_{\theta}^{n}\right)$ cannot be unitarily equivalent by an inner automorphism of the algebra.

We also compute the action of unitary transformations induced by some outer automorphisms on the spin structures. These are the action of $\operatorname{SL}(2, \mathbb{Z})$ on the noncommutative 2 -torus and the flip automorphism on the noncommutative $n$-torus for $n>2$.

The set of $\theta$ of measure 0 in Theorem $\mathrm{B}$ is determined by some Diophantine approximation conditions given in [5], and includes the $\theta$ with only rational entries.

In addition to unitary equivalences of real spectral triples, one could also look at Morita equivalences of real spectral triples [37], Chapter 7.2, even though it is not a true equivalence since it is not symmetric in general, see [12], Remark 1.143. It would be interesting to study Morita equivalences of spin structures on noncommutative tori, especially since all Morita equivalences of the algebra of functions on the smooth noncommutative tori are known [32], [20], Theorem 1.1. We hope to return to this question in the future.

\section{Definition of a real spectral triple}

Since there are various, slightly different, definitions of real spectral triples, cf. [9], p. 159-162, [37], Chapter 3, [22], Chapter 10, and we need to refer to the conditions unambiguously, we will explicitly state the definition of real spectral triple we use. For the definition of an equivariant spectral triple, see Section 2.1. A spin structure on a manifold $M$, of dimension $n$, is a nontrivial double cover of the principal $\operatorname{SO}(n)$ bundle of orthogonal frames of the tangent bundle TM [26], Chapter II.1. After [10], we know that a real spectral triple is the right noncommutative geometry analog of a spin structure on a manifold.

For a real spectral triple we have the following data:

- a unital Fréchet algebra $\mathcal{A}, *$-isomorphic to a proper dense subalgebra of a $\mathrm{C}^{*}$-algebra which is stable with respect to the holomorphic functional calculus;

- a separable Hilbert space $\mathscr{H}$ with a representation of $\mathcal{A}$ acting as bounded operators;

- an unbounded self-adjoint operator $D$, called 'Dirac operator';

- an antilinear isometry $J$ of $\mathscr{H}$ onto itself, called 'reality operator';

- an integer $n \geq 0$, called 'dimension'; 
- if $n$ is even, a self-adjoint unitary operator $\Gamma$ of $\mathscr{H}$ onto itself such that $\Gamma^{2}=\mathrm{Id}$, called 'grading operator'. We call the spectral triple even in this case.

These objects also satisfy the Conditions 1 to 9 in order for them to be called a real spectral triple of dimension $n$.

Condition 1 (Compact resolvent). The Dirac operator $D$ has compact resolvent, that is, $D$ has finite dimensional kernel and $D^{-1}$ (defined on the orthogonal complement of the kernel) is a compact operator. Furthermore, for all $a \in \mathcal{A},[D, \pi(a)]$ is a bounded operator.

Condition 2 (Grading operator). If $n$ is even, the $\mathbb{Z}_{2}$ grading operator $\Gamma$ splits the Hilbert space $\mathscr{H}$ into $\mathscr{H}^{+}$and $\mathscr{H}^{-}$, where $\mathscr{H}^{ \pm}$is the $( \pm)$eigenspace of $\Gamma$. The operator $D$ is odd with respect to this operator, $D \Gamma=-\Gamma D$, and the representation $\pi$ of $\mathcal{A}$ on $\mathscr{H}$ is even, so we can write

$$
\pi(a)=\left(\begin{array}{ll}
a & 0 \\
0 & a
\end{array}\right), \quad D=\left(\begin{array}{cc}
0 & D^{-} \\
D^{+} & 0
\end{array}\right)
$$

where $D^{+}$and $D^{-}$are adjoint to each other.

Condition 3. The operators $J, D$ and $\Gamma$ satisfy the commutation relations from Table 1, and the operator $J$ is unitary: $J^{\dagger}=J^{-1}$.

Table 1. Signs of the spectral triple.

\begin{tabular}{|c|c|c|c|c|c|c|c|c|c|c|c|}
\hline \multicolumn{4}{|c|}{$n \bmod 8$} & 0 & 1 & 2 & 3 & 4 & 5 & 6 & \\
\hline$J^{2}$ & $=$ & $\pm \mathrm{Id}$ & $\left(\epsilon_{J}\right)$ & + & + & - & - & - & - & + & \\
\hline$J D$ & $=$ & $\pm D J$ & $\left(\epsilon_{D}\right)$ & + & - & + & + & + & - & + & \\
\hline$J \Gamma$ & $=$ & $\pm \Gamma J$ & $\left(\epsilon_{\Gamma}\right)$ & + & & - & & + & & - & \\
\hline
\end{tabular}

Condition 4 (Dimension). The eigenvalues $\mu_{k}$ of $|D|^{-1}$, arranged in decreasing order, grow asymptotically,

$$
\mu_{k}=\mathcal{O}\left(k^{-n}\right),
$$

where $n$ is an integer called the dimension.

Condition 5 (First order condition). For all $a, b \in \mathcal{A}$ the following commutation relations hold:

$$
\begin{aligned}
{\left[a, J b^{*} J^{\dagger}\right] } & =0 . \\
{\left[[D, a], J b^{*} J^{\dagger}\right] } & =0 .
\end{aligned}
$$


We will write $b^{0}=J b^{*} J^{\dagger}$. The above formulas establish that the opposite algebra:

$$
\mathcal{A}^{\mathrm{o}}=\left\{a^{\mathrm{o}}=J^{\dagger} a^{*} J \mid a \in \mathcal{A}\right\},
$$

lies within the commutator of $\mathcal{A}$.

Recall that a Hochschild $k$-chain is defined as an element $c$ of $C_{k}(M, \mathcal{A})=$ $M \otimes \mathcal{A}^{\otimes k}$, with $M$ a bimodule over $\mathcal{A}$. A boundary map $b: C_{k} \rightarrow C_{k-1}$ is defined as

$$
\begin{aligned}
b & =\sum_{i=0, k}(-1)^{i} d_{i}, \\
d_{0}\left(m \otimes a_{1} \otimes \cdots \otimes a_{k}\right) & =m a_{1} \otimes a_{2} \cdots \otimes a_{k}, \\
d_{i}\left(m \otimes a_{1} \otimes \cdots \otimes a_{k}\right) & =m \otimes a_{1} \otimes \cdots \otimes a_{i} a_{i+1} \otimes \cdots \otimes a_{k}, \\
d_{k}\left(m \otimes a_{1} \otimes \cdots \otimes a_{k}\right) & =a_{k} m \otimes a_{1} \otimes \cdots \otimes a_{k-1} .
\end{aligned}
$$

Since $b^{2}=0$, this makes $C_{k}(M, \mathcal{A})$ into a chain complex.

Condition 5 gives a representation of Hochschild $k$-chains $C_{k}\left(\mathcal{A}, \mathcal{A} \otimes \mathcal{A}^{\circ}\right)$ on $\mathscr{H}$ by

$$
\pi_{D}\left(\left(a \otimes b^{\mathrm{o}}\right) \otimes a_{1} \otimes \cdots \otimes a_{k}\right)=a b^{\mathrm{o}}\left[D, a_{1}\right] \ldots\left[D, a_{k}\right]
$$

Condition 6 (Orientability). There is a Hochschild cycle $c \in Z_{n}\left(\mathcal{A}, \mathcal{A} \otimes \mathcal{A}^{\circ}\right)$ such that $\pi_{D}(c)=\Gamma$ when $n$ is even, and $\pi_{D}(c)=$ Id when $n$ is odd.

Condition 7 (Regularity). For all $a \in \mathcal{A}$, both $a$ and $[D, a]$ belong to the domain of smoothness $\bigcap_{k=1}^{\infty} \operatorname{Dom}\left(\delta^{k}\right)$, where the derivation $\delta$ is given by $\delta(T)=[|D|, T]$ with $|D|=\sqrt{D^{*} D}$.

Condition 8 (Finiteness). The space of smooth vectors $\mathscr{H}^{\infty}=\bigcap_{k=1}^{\infty} \operatorname{Dom}\left(D^{k}\right)$ is a finitely generated projective left $\mathcal{A}$ module. Also, there is a Hermitian pairing $(\eta \mid \xi)$ on this module given by

$$
\langle\eta, \xi\rangle=f(\eta \mid \xi)|D|^{-n},
$$

where $f$ is the noncommutative integral (defined for example in [37], Chapter 5).

Condition 9 (Poincaré duality). The Fredholm index of the operator $D$ yields a nondegenerate intersection form on the $K$-theory ring of the algebra $\mathcal{A} \otimes \mathcal{A}^{0}$.

Finally, we restrict our attention to irreducible spectral triples, that is, the only operators commuting with the action of the algebra and $D$ are the scalars. In the case $\mathcal{A}$ is commutative, this is equivalent to demanding the manifold is connected, see [9], Remark 6 on p. 163. 
2.1. Equivariant spectral triples. There are different candidates for the notion of symmetries of noncommutative geometries. One obvious candidate is the group of automorphisms of the algebra $\mathcal{A}$, but for noncommutative algebras this group can be very small, while it seems that there should be more symmetries available. An attempt to enlarge this group of symmetries in an interesting way is the notion of equivariant spectral triples. Equivariant spectral triples were introduced in [34]. One describes symmetries of spectral triples in the form of Hopf algebras. In this paper we are interested in $n$-dimensional spectral triples which are equivariant with respect to a Hopf algebra with $n$ different commuting derivations. This, together with the irreducibility condition, is the analog of a connected compact homogeneous space in commutative geometry.

In the context of Hopf algebras, we shall use Sweedler's notation for the coproduct: $\Delta h=h_{(1)} \otimes h_{(2)}$. See for example [24], Chapter 3, for an introduction to Hopf algebras and some standard notation. An equivariant real spectral triple is a real spectral triple $(\mathcal{A}, \mathcal{H}, D, J)$ together with a Hopf algebra $H$, with multiplication $\mu$, unit $\eta$, comultiplication $\Delta$, counit $\epsilon$ and antipode $S$, and an antilinear involution * making $H$ into a $*$-algebra such that

$$
\Delta h^{*}=(\Delta h)^{* \otimes *}, \quad \epsilon\left(h^{*}\right)=\overline{\epsilon(h)}, \quad(S \circ *)^{2}=\text { Id } .
$$

Recall that an $H$-module algebra is an algebra $A$ with a complex linear representation $\rho$ of $H$ on $A$ such that $A$ is a linear space, and $\rho$ respects the algebra structure

$$
\rho(h)\left(a_{1} a_{2}\right)=\left(\rho\left(h_{(1)}\right) a_{1}\right)\left(\rho\left(h_{(2)}\right) a_{2}\right)
$$

for all $h \in H, a_{1}, a_{2} \in A$. When $A$ is an $H$-module algebra we define an equivariant (left, right) $A$-module to be a (left, right) $A$-module $M$ such that

$$
\rho_{M}(h)(a m)=\left(\rho_{A}\left(h_{(1)}\right) a\right)\left(\rho_{M}\left(h_{(2)}\right) m\right)
$$

for all $h \in H, a \in A, m \in M$. The objects of the equivariant real spectral triple transform in a compatible way under the action of the Hopf algebra:

- The algebra $\mathcal{A}$ is an $H$-module algebra.

- There is a dense subspace $V \subset \mathscr{H}$ such that $V$ is an equivariant left $\mathcal{A}$-module.

- For every $h \in H$, the Dirac operator is equivariant, $[D, h]=0$ on the (dense) intersection of the domain of $D$ and $V$.

- The action of $H^{\text {op }}$ is well defined on the opposite algebra $\mathcal{A}^{\circ}$ via the equality $J^{-1} h J=(\mathrm{Sh})^{*}$.

- If the spectral triple is even, then $[\Gamma, h]=0$.

In the case of equivariance with respect to a torus action, we take the universal enveloping algebra $U\left(\mathrm{t}^{n}\right)$ of the familiar Lie algebra $\mathrm{t}^{n}$ of the $n$-torus. This means that we have a basis of derivations $\delta_{i}$, with a representation $\rho$ on $\mathscr{H}$ such that for 
$a \in \mathcal{A}\left(\mathbb{\nabla}_{\theta}^{n}\right), v \in \mathscr{H}:$

$$
\begin{aligned}
\delta_{i} \delta_{j} & =\delta_{j} \delta_{i}, \\
\Delta\left(\delta_{i}\right) & =\delta_{i} \otimes \mathrm{Id}+\mathrm{Id} \otimes \delta_{i}, \\
\rho\left(\delta_{i}\right) \pi(a) v & =\left(\pi\left(\delta_{i} a\right)+\pi(a)\right) v, \\
\rho\left(\delta_{i}\right) D v & =D \rho\left(\delta_{i}\right) v, \\
\rho\left(\delta_{i}\right) J v & =-J \rho\left(\delta_{i}\right)^{*} v .
\end{aligned}
$$

\section{Outline of the classification}

The outline of the proof of Theorem A is as follows. First in Section 4 we determine the action of the algebra on the Hilbert space such that the equivariance condition is met. This action is already well known, but we derive it to show that there are no other possibilities. In Section 5 we move to real spectral triples and determine possible forms of the reality operator $J$ by considering the anti-isomorphism $\mathcal{A}\left(\mathbb{T}_{\theta}^{n}\right) \mapsto$ $\mathcal{A}\left(\mathbb{T}_{\theta}^{n}\right)^{\circ}$ and the equivariance condition (3e). We find several possible families of real spectral triples, only one of which consists of isospectral deformations of spin structures on the commutative torus. In Section 6 we determine the classes of possible Dirac operators for each candidate family of real spectral triples using equivariance of the Dirac operator and the first-order condition, and show that only the isospectral deformation family is compatible with the compact resolvent condition.

In Section 7 we determine that the parameters $\tau$ in the Dirac operators must be linearly independent vectors spanning $\mathbb{R}^{n}$, using the Hochschild homology condition and earlier results on the Hochschild homology on noncommutative tori. The last step in the classification is done in Section 8, where we use the spin manifold reconstruction theorem to show that the Dirac operator is really a Dirac operator in the sense of spin geometry.

After the classification, we give in Section 9 an explicit description of the constructed real spectral triples on the noncommutative torus.

Finally, in Section 10, we study unitary equivalences of the real spectral triples, and show that unitary equivalences induced by inner automorphisms of the algebra do not change the spin structure for almost all $\theta$. When $n=2$ we show that the known outer automorphisms do change the spin structure if the real spectral triple is an isospectral deformation of a nontrivial spin structure. When $n>2$, we show that the flip automorphism cannot change the spin structure.

\section{Hilbert space and algebra}

We look for possible equivariant representations of the algebra of functions of the smooth noncommutative torus and give a basis of the Hilbert space $\mathscr{H}$ for which 
equivariance is obvious. We do not use any special conditions from the definition of a real spectral triple, except that the Hilbert space should be separable.

We denote the noncommutative torus, or more precisely, the algebra of continuous functions on the noncommutative $n$-torus, by $A\left(\mathbb{T}_{\theta}^{n}\right)$, where $\theta$ is an antisymmetric real $(n \times n)$-matrix. As Hopf algebra symmetry for which the algebra representation must be equivariant we take the Hopf algebra generated by $n$ independent commuting elements $\delta_{1}, \ldots, \delta_{n}$.

The algebra of smooth or continuous functions on the noncommutative torus is generated by unitary elements $U_{e_{1}}, \ldots, U_{e_{n}}$ such that

$$
U_{e_{k}} U_{e_{l}}=\exp \left(2 \pi i \theta_{k l}\right) U_{e_{l}} U_{e_{k}},
$$

with $\theta_{k l}$ the component of the matrix at position $(k, l)$. As a short-hand notation, we will write

$$
e(\cdot)=\exp (2 \pi i \cdot)
$$

The Hopf algebra action of our basis elements on the unitary generators is [34]: $\delta_{i} U_{e_{j}}=U_{e_{j}}$ if $i=j$, and 0 otherwise. If we interpret the $e_{j}$ as the $j$-th basis vector of $\mathbb{Z}^{n}$, we can write more generally unitary elements of the algebra in the form

$$
U_{\mathbf{x}}=e\left(\frac{1}{2} \sum_{k>j} x_{j} \theta_{j k} x_{k}\right)\left(U_{e_{1}}\right)^{x_{1}}\left(U_{e_{2}}\right)^{x_{2}} \ldots\left(U_{e_{n}}\right)^{x_{n}}
$$

for $\mathbf{x}=\sum x_{j} e_{j}$. The Fréchet algebra of smooth functions on the noncommutative torus is a dense subalgebra of this algebra.

Just as for the algebra, we will write $\delta_{\mathbf{x}}$ for the derivation given by

$$
\delta_{\mathbf{x}}=\delta_{x_{1}} \delta_{x_{2}} \ldots \delta_{x_{n}} .
$$

From the definitions, it is immediate that

$$
\delta_{\mathbf{x}} U_{\mathbf{y}}=(\mathbf{x} \cdot \mathbf{y}) U_{\mathbf{y}},
$$

where $(\mathbf{x} \cdot \mathbf{y})$ is the standard inner product on $\mathbb{Z}^{n}$.

Now we look for a Hilbert space $\mathscr{H}_{0}$ which is an equivariant left $A_{\theta}$-module. As a basis of $\mathscr{H}_{0}$ we choose mutual eigenvectors $e_{\mu}$ of the derivations,

$$
\rho\left(\delta_{i}\right) e_{\boldsymbol{\mu}}=\mu_{i} e_{\boldsymbol{\mu}},
$$

where the $\mu$ form a countable subset of $\mathbb{R}^{n}$.

In order for the spectral triple to be a noncommutative torus, we demand that the action of the algebra is equivariant with respect to a torus action, as in equation (3c). Written out for the unitary generators $U_{\mathbf{x}}$, we see that

$$
\pi_{0}\left(U_{\mathbf{x}}\right) e_{\boldsymbol{\mu}}=u_{\mathbf{x}, \boldsymbol{\mu}} e_{\boldsymbol{\mu}+\mathbf{x}},
$$

with $u_{\mathrm{x}, \boldsymbol{\mu}} \in \mathbb{C}$ to be determined. Thus for the minimal irreducible equivariant representation the $\boldsymbol{\mu}$ will lie in a translate of a lattice,

$$
\mathscr{H}_{0}=\bigoplus_{\mathbf{m} \in \mathbb{Z}^{n}} \mathscr{H}_{\boldsymbol{\mu}_{0}+\mathbf{m}},
$$


where each $\mathscr{H}_{\boldsymbol{\mu}_{0}+\mathbf{m}} \cong \mathbb{C}$. There are no restrictions yet on $\boldsymbol{\mu}_{0}$, these will be determined later.

Since the $U_{\mathbf{x}}$ should be unitary, we have that

$$
\left\langle e_{\boldsymbol{v}}, u_{\mathbf{x}, \boldsymbol{\mu}} e_{\boldsymbol{\mu}+\mathbf{x}}\right\rangle=\left\langle u_{-\mathbf{x}, \boldsymbol{v}} e_{\boldsymbol{v}-\mathbf{x}}, e_{\boldsymbol{\mu}}\right\rangle \Rightarrow u_{\mathbf{x}, \boldsymbol{\mu}} \delta_{\boldsymbol{v}, \boldsymbol{\mu}+\mathbf{x}}=\overline{u_{-\mathbf{x}, \boldsymbol{v}}} \delta_{\boldsymbol{v}-\mathbf{x}, \boldsymbol{\mu}} .
$$

Finally the definition of $U_{\mathbf{x}}$ in terms of $U_{i}$ gives the relations

$$
u_{\mathbf{x}+\mathbf{y}, \boldsymbol{\mu}}=e\left(\frac{1}{2} \mathbf{x} \cdot \theta \mathbf{y}\right) u_{\mathbf{x}, \boldsymbol{\mu}+\mathbf{y}} u_{\mathbf{y}, \boldsymbol{\mu}} .
$$

Lemma 1. Up to unitary transformations of $\mathscr{H}$ any unitary equivariant representation of $\mathcal{A}\left(\mathbb{T}_{\theta}^{n}\right)$ on $\mathscr{H}$ is given by

$$
\pi_{0}^{\mathbf{A}}\left(U_{\mathbf{x}}\right) e_{\boldsymbol{\mu}}=e\left(\frac{1}{2} \mathbf{x} \cdot \mathbf{A} \mathbf{x}+\mathbf{x} \cdot \mathbf{A} \boldsymbol{\mu}\right) e_{\boldsymbol{\mu}+\mathbf{x}},
$$

with $\mathbf{A}$ any $(n \times n)$-matrix such that $\mathbf{A}-\mathbf{A}^{t}=\theta$.

Since the representations $\pi_{0}^{\mathbf{A}}$, given by different matrices $\mathbf{A}$ such that $\mathbf{A}-\mathbf{A}^{t}=\theta$ are equivalent, we drop the $\mathbf{A}$ from the notation, and just write $\pi_{0}$ for a representation defined by equation (7).

Proof. It is clear that for any matrix $\mathbf{A}$ the representation given above satisfies the relations (5) and (6). Given two representations $\pi$ and $\pi^{\prime}$ satisfying the equivariance condition (3c) we can write any element $w \in \mathscr{H}$ as a unique sum $\sum_{\mathbf{x} \in \mathbb{Z}^{n}} \lambda_{\mathbf{x}} \pi\left(U_{\mathbf{x}}\right) e_{0}$

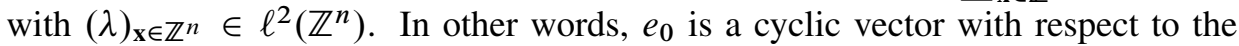
algebra action. Now construct an operator $T: \mathcal{H} \rightarrow \mathscr{H}$ by setting $T e_{0}=e_{0}$ and extending by

$$
T w=T \sum_{\mathbf{x} \in \mathbb{Z}^{n}} \lambda_{\mathbf{x}} \pi\left(U_{\mathbf{x}}\right) e_{0}=\sum_{\mathbf{x} \in \mathbb{Z}^{n}} \lambda_{\mathbf{x}} \pi^{\prime}\left(U_{\mathbf{x}}\right) e_{0} .
$$

This is well defined if $\pi$ and $\pi^{\prime}$ are representations of the same algebra $\mathcal{A}\left(\mathbb{T}_{\theta}^{n}\right)$ since they satisfy the same algebra relation (6), and it is an invertible map on $\mathscr{H}$ because both $\pi\left(U_{\mathbf{x}}\right) e_{0}$ and $\pi^{\prime}\left(U_{\mathbf{x}}\right) e_{0}$ span the Hilbert space if we take all $\mathbf{x} \in \mathbb{Z}^{n}$. By construction $T^{-1} \pi^{\prime}\left(U_{\mathbf{x}}\right) T=\pi\left(U_{\mathbf{x}}\right)$, and it is a unitary transformation because we can calculate

$$
\begin{aligned}
\langle T v, T w\rangle & =\sum_{\mathbf{x}, \mathbf{y} \in \mathbb{Z}^{n}} \bar{\lambda}_{\mathbf{x}} \mu_{\mathbf{y}}\left\langle\pi^{\prime}\left(U_{\mathbf{x}}\right) e_{0}, \pi^{\prime}\left(U_{\mathbf{y}}\right) e_{0}\right\rangle \\
& =\sum_{\mathbf{x}-\mathbf{y}=0} \bar{\lambda}_{\mathbf{x}} \mu_{\mathbf{y}}\left\langle\pi^{\prime}\left(U_{\mathbf{x}}\right) e_{0}, \pi^{\prime}\left(U_{\mathbf{y}}\right) e_{0}\right\rangle \\
& =\sum_{\mathbf{x} \in \mathbb{Z}^{n}} \bar{\lambda}_{\mathbf{x}} \mu_{-\mathbf{x}} \\
& =\langle v, w\rangle,
\end{aligned}
$$

since $\left\langle e_{\mathbf{x}}, e_{\mathbf{y}}\right\rangle=0$ if $\mathbf{x} \neq \mathbf{y}$, and the representations $\pi$ and $\pi^{\prime}$ are unitary.

There might be more unitary equivalences of the algebra, a question which we explore in Section 10, but for now we were only interested in possible representations of the algebra. 


\section{Reality operator}

In this section, we derive conditions on the equivariant reality operator $J$, to give us a real spectral triple. We will only consider conditions following from relations between $\mathcal{A}, \mathscr{H}$ and $J$ and the equivariance condition (3e). No use is made of the Dirac operator in this section.

The equivariance condition for $J$ is given in (3e):

$$
\rho(l) J v=-J \rho\left(l^{*}\right) v
$$

for $v \in \mathscr{H}$ and $l$ an element of the Hopf algebra of the symmetries of the noncommutative $n$-torus. For our basic derivations $\delta_{i}$, we have $\delta_{i}^{*}=\delta_{i}$, so this just means

$$
\delta_{i} J e_{\boldsymbol{\mu}}=-\mu_{i} J e_{\boldsymbol{\mu}}
$$

for all $i$. Since we are working with the representation given in (4), we see that $J$ must map an element $e_{\mu}$ of the basis to $e_{-\mu}$.

The Tomita involution $J_{0}(a)=a^{*}$ [35] gives a commuting representation, but it does not follow the commutation relations in Table 1, so we will have to enlarge our Hilbert space. Define

$$
\mathscr{H}:=\bigoplus_{j \in I} \mathscr{H}_{j},
$$

with each $\mathscr{H}_{j}$ as in (4) and $I$ an index set. Every nondegenerate representation of an involutive Banach algebra is a direct sum of cyclic representations [36], Proposition I.9.17, and because of the equivariance condition (3c), we get that the only cyclic representations we can consider are the ones given by Lemma 1 . We write basis vectors of this Hilbert space as $e_{\boldsymbol{\mu}, j}$ with $\boldsymbol{\mu} \in \mathbb{Z}^{n}$ and $j \in I$.

For different $j \in I$, lattices spanned by $\mu$ could a priori be shifted by a different amount, satisfying (9), but we will see in Section 6 that this cannot be the case if the spectral triple is irreducible.

We look for an antilinear operator $J$ such that $J^{2}=\epsilon_{J}$ Id, with signs as in Table 1 , and such that, for every $a \in \mathcal{A}\left(\mathbb{T}_{\theta}^{n}\right), J a^{*} J^{-1}$ commutes with all $b \in \mathcal{A}\left(\mathbb{T}_{\theta}^{n}\right)$ satisfying equation (1). The image of $\mathcal{A}\left(\mathbb{T}_{\theta}^{n}\right)$ under the isomorphism $a \mapsto J a^{*} J^{-1}$ is denoted by $\mathcal{A}\left(\mathbb{T}_{\theta}^{n}\right)^{\mathrm{o}}$. We write $U_{\mathbf{x}}^{\mathrm{o}}$ for the image of $U_{\mathbf{x}}$.

Firstly, equation (8) has as a consequence that $J$ acts as follows on elements of the basis $e_{\mu, j}$ :

$$
J e_{\mu, j}=\sum a_{k j}(\boldsymbol{\mu}) e_{-\mu, k} .
$$

We can thus write $J e_{\boldsymbol{\mu}, j}=\Lambda(-\boldsymbol{\mu}) J_{0} e_{\boldsymbol{\mu}, j}$, with $\Lambda(-\boldsymbol{\mu})$ some unitary or skew unitary bounded linear functional and $J_{0}$ an antilinear diagonal operator:

$$
J_{0} a e_{\mu, j}=a^{*} e_{-\mu, j} .
$$

If we apply $J$ twice, we should get $\epsilon_{J}$ Id, which can be written as

$$
J \cdot J e_{\mu, j}=J \Lambda(-\mu) e_{-\mu, j}=\Lambda(\mu) \Lambda^{*}(-\mu) e_{-\mu, j},
$$


and so we see that

$$
\Lambda(\mu) \Lambda(-\mu)^{*}=\epsilon_{J} \mathrm{Id}
$$

By applying $J$ on a unitary generator $U_{\mathbf{x}}$ of $\mathcal{A}_{\theta}$ we get the following condition on $\Lambda(\mu)$ :

Lemma 2. The map $a \mapsto J a^{*} J^{\dagger}$ is an isomorphism into the commutant if and only if for all $\mathbf{x}, \mathbf{y} \in \mathbb{Z}^{n}$ :

$$
\Lambda(\mathbf{x}+\mathbf{y})=e(\mathbf{x} \cdot \mathbf{A y}+\mathbf{y} \cdot \mathbf{A x}) \Lambda(\mathbf{x}) \Lambda(0)^{\dagger} \Lambda(\mathbf{y})
$$

where $J e_{\boldsymbol{\mu}, j}=\Lambda(-\mu) J_{0} e_{\boldsymbol{\mu}, j}$, with $J_{0}$ the Tomita involution, and

$$
\Lambda(\mathbf{x}) \Lambda(\mathbf{x})^{\dagger}=\mathrm{Id}, \quad \Lambda(\mathbf{x}) \Lambda(-\mathbf{x})^{*}=\epsilon_{J} \mathrm{Id} .
$$

As a consequence we have

$$
U_{\mathbf{x}}^{\mathrm{o}} e_{\boldsymbol{\mu}, j}=e\left(\boldsymbol{\mu} \cdot \mathbf{A x}+\frac{1}{2} \mathbf{x} \cdot \mathbf{A} \mathbf{x}\right) \Lambda^{\prime}(\mathbf{x}) \Lambda(0)^{\dagger} e_{\boldsymbol{\mu}+\mathbf{x}, j},
$$

where $\Lambda(\boldsymbol{\mu})=e(\boldsymbol{\mu} \cdot \mathbf{A} \boldsymbol{\mu}) \Lambda^{\prime}(\boldsymbol{\mu})$, and $\Lambda^{\prime}(\boldsymbol{\mu})_{i j}=c_{i j} e\left(\boldsymbol{\phi}_{i j}(\boldsymbol{\mu})\right)$ with $c_{i j} \in \mathbb{C}$ and $\phi_{i j}: \mathbb{R}^{n} \rightarrow \mathbb{R}$ such that $\sum_{j \in I} c_{i j} e\left(\boldsymbol{\phi}_{i j}(-\boldsymbol{\mu})\right) c_{j k}^{*} e\left(-\boldsymbol{\phi}_{i j}(\boldsymbol{\mu})\right)=\epsilon_{J} \delta_{i k}$.

Proof. First we calculate $U_{\mathbf{x}}^{\mathrm{o}}=J U_{\mathbf{x}}^{*} J^{\dagger}$ using equation (11):

$$
\begin{aligned}
U_{\mathbf{x}}^{\mathrm{o}} e_{\boldsymbol{\mu}, j} & =J U_{\mathbf{x}}^{*} J^{\dagger} e_{\boldsymbol{\mu}, j} \\
& =J U_{\mathbf{x}}^{*} \Lambda(\boldsymbol{\mu})^{t} e_{-\boldsymbol{\mu}, j} \\
& =J e\left(\mathbf{x} \cdot \mathbf{A} \boldsymbol{\mu}+\frac{1}{2} \mathbf{x} \cdot \mathbf{A x}\right) \Lambda(\boldsymbol{\mu})^{t} e_{-\boldsymbol{\mu}-\mathbf{x}, j} \\
& =\Lambda(\boldsymbol{\mu}+\mathbf{x}) e\left(-\mathbf{x} \cdot \mathbf{A} \boldsymbol{\mu}-\frac{1}{2} \mathbf{x} \cdot \mathbf{A x}\right) \Lambda(\boldsymbol{\mu})^{\dagger} e_{\boldsymbol{\mu}+\mathbf{x}, j}
\end{aligned}
$$

We compute the commutator $\left[U_{\mathbf{y}}, U_{\mathbf{x}}^{\mathrm{o}}\right]$ :

$$
\begin{aligned}
U_{\mathbf{y}} U_{\mathbf{x}}^{\mathrm{o}} e_{\boldsymbol{\mu}, j}= & e\left(\mathbf{y} \cdot \mathbf{A}\left(\boldsymbol{\mu}+\mathbf{x}+\frac{1}{2} \mathbf{y}\right)-\mathbf{x} \cdot \mathbf{A}\left(\boldsymbol{\mu}+\frac{1}{2} \mathbf{x}\right)\right) \Lambda(\boldsymbol{\mu}+\mathbf{x}) \Lambda^{\dagger}(\boldsymbol{\mu}) e_{\boldsymbol{\mu}+\mathbf{x}+\mathbf{y}, j} \\
U_{\mathbf{x}}^{\mathrm{o}} U_{\mathbf{y}} e_{\boldsymbol{\mu}, j}= & e\left(-\mathbf{x} \cdot \mathbf{A}\left(\boldsymbol{\mu}+\mathbf{y}+\frac{1}{2} \mathbf{x}\right)\right. \\
& \left.+\mathbf{y} \cdot \mathbf{A}\left(\boldsymbol{\mu}+\frac{1}{2} \mathbf{y}\right)\right) \Lambda(\boldsymbol{\mu}+\mathbf{x}+\mathbf{y}) \Lambda^{\dagger}(\boldsymbol{\mu}+\mathbf{y}) e_{\boldsymbol{\mu}+\mathbf{x}+\mathbf{y}, j}
\end{aligned}
$$

If the commutator vanishes, we see that by canceling common terms we must have

$$
e(\mathbf{y} \cdot \mathbf{A x}) \Lambda(\boldsymbol{\mu}+\mathbf{x}) \Lambda^{\dagger}(\boldsymbol{\mu})=e(-\mathbf{x} \cdot \mathbf{A y}) \Lambda(\boldsymbol{\mu}+\mathbf{x}+\mathbf{y}) \Lambda^{\dagger}(\boldsymbol{\mu}+\mathbf{y})
$$

This has as a consequence that $\Lambda(\mathbf{x})_{i j}$ consists of $e\left(f_{i j}(x)\right)$ with $f_{i j}(x)$ a function of the form $\mathbf{x} \cdot \mathbf{B}_{i j} \mathbf{x}+\boldsymbol{\phi}_{i j}(\mathbf{x})+v_{i j}$. We see that the quadratic part must be the same for each component, and that $\mathbf{B}_{i j}=\mathbf{A}$. The constant part $v_{i j}$ can be absorbed into by unitary transformation. We can thus write

$$
\Lambda(\mathbf{x})=e(\mathbf{x} \cdot \mathbf{A x}) \Lambda^{\prime}(\mathbf{x}),
$$


where $\Lambda^{\prime}(\mathbf{x})$ consists of functions $c_{i j} e\left(\phi_{i j}(\mathbf{x})\right)$ such that $\Lambda^{\prime}(\mathbf{x})=\epsilon_{J} \Lambda^{\prime}(-x)^{t}$ and $\Lambda^{\prime}(\mathbf{x}) \Lambda^{\prime}(\mathbf{x})^{\dagger}=$ Id. If we then calculate $U_{\mathbf{x}}^{\mathrm{o}}$, we obtain

$$
\begin{aligned}
U_{\mathbf{x}}^{\mathrm{o}} e_{\boldsymbol{\mu}}= & \Lambda(\boldsymbol{\mu}+\mathbf{x}) e\left(-\mathbf{x} \cdot \mathbf{A} \boldsymbol{\mu}-\frac{1}{2} \mathbf{x} \cdot \mathbf{A} \mathbf{x}\right) \Lambda(\boldsymbol{\mu})^{\dagger} e_{\boldsymbol{\mu}+\mathbf{x}, j} \\
= & e((\boldsymbol{\mu}+\mathbf{x}) \cdot \mathbf{A}(\boldsymbol{\mu}+\mathbf{x})-\mathbf{x} \cdot \mathbf{A} \boldsymbol{\mu} \\
& \left.-\frac{1}{2} \mathbf{x} \cdot \mathbf{A} \mathbf{x}-\boldsymbol{\mu} \cdot \mathbf{A} \boldsymbol{\mu}\right) \Lambda^{\prime}(\boldsymbol{\mu}+\mathbf{x}) \Lambda^{\prime}(\boldsymbol{\mu})^{\dagger} e_{\boldsymbol{\mu}+\mathbf{x}, j} \\
= & e\left(\boldsymbol{\mu} \cdot \mathbf{A} \mathbf{x}+\frac{1}{2} \mathbf{x} \cdot \mathbf{A x}\right) \Lambda^{\prime}(\boldsymbol{\mu}+\mathbf{x}) \Lambda^{\prime}(\boldsymbol{\mu})^{\dagger} e_{\boldsymbol{\mu}+\mathbf{x}, j} \\
= & e\left(\boldsymbol{\mu} \cdot \mathbf{A} \mathbf{x}+\frac{1}{2} \mathbf{x} \cdot \mathbf{A x}\right) \Lambda^{\prime}(\mathbf{x}) \Lambda^{\prime}(0)^{\dagger} e_{\boldsymbol{\mu}+\mathbf{x}, j} .
\end{aligned}
$$

By definition (13) of $\Lambda^{\prime}$, we have $\Lambda^{\prime}(0)=\Lambda(0)$, so this is exactly equation (12).

\section{Dirac operator}

The remaining piece of the real spectral triple is the Dirac operator. In this section, using the results from the previous section, the equivariance condition (3d), and Conditions 1 and 5, we derive conditions for the Dirac operator $D$. We see that for different forms of $\Lambda$ as given in Lemma 2 only the form $\Lambda^{\prime}(\mathbf{x})^{2}=$ Id can lead to isospectral deformations of spin structures on the commutative $n$-torus. By applying the compact resolvent condition of Condition 1, we show that this is the only possibility compatible with the definition of noncommutative geometry.

An equivariant Dirac operator $D$ commutes with the basic derivations $\delta_{i}$ as described in (3d):

$$
D e_{\boldsymbol{\mu}, j}=\sum_{k \in I} d_{\boldsymbol{\mu}, j}^{k} e_{\boldsymbol{\mu}, k} .
$$

Since $D$ should be self-adjoint, we have $d_{\boldsymbol{\mu}, j}^{k}=\left(d_{\boldsymbol{\mu}, k}^{j}\right)^{*}$. We will write

$$
D e_{\mu, j}=D(\mu) e_{\mu, j} .
$$

This means that $D(\boldsymbol{\mu})$ is the operator $D$ restricted to the eigenspace of derivations with eigenvalue $\boldsymbol{\mu}$. This is well defined due to the equivariance condition (3d).

Given an element $\boldsymbol{\mu}$ in a shifted lattice $\widetilde{\mathbb{Z}}^{n}$, we define the Hilbert space $\mathscr{H}_{\boldsymbol{\mu}}$ as the span of eigenvectors $v_{j}$ of the basic derivations $\delta_{i}$ such that $\delta_{i} v_{j}=\mu_{i} v_{j}$ for each $i$. As a consequence of the equivariance of the Dirac operator and the irreducibility condition, all the $\mu$ must lie in the same lattice, by the following argument.

Between two Hilbert spaces $\mathscr{H}_{\boldsymbol{\mu}}$ and $\mathscr{H}_{\boldsymbol{v}}$ we have an isometry if $\boldsymbol{\mu}-\boldsymbol{v} \in \mathbb{Z}^{n}$, given by the unitary element $U_{\boldsymbol{\mu}-\boldsymbol{v}}$ of the algebra. Now consider the projector $P_{\boldsymbol{\mu}}$ that is Id on $\mathscr{H}_{\boldsymbol{v}}$ for which $\boldsymbol{\mu}-\boldsymbol{v} \in \mathbb{Z}^{n}$, and 0 otherwise. This projection clearly commutes with the algebra, and, because of (14), also with the Dirac operator. If the spectral triple is irreducible, only scalars may commute with both the algebra and the 
Dirac operator, so $P_{\boldsymbol{\mu}}$ is the identity on the whole Hilbert space $\mathscr{H}$, hence all lattices are shifted by the same vector $\epsilon$. Because of (9), we can then conclude that $\epsilon$ consists of elements which are either 0 or $\frac{1}{2}$.

From the first order condition in equation (2), we deduce:

Lemma 3. An equivariant Dirac operator $D$ which satisfies the first order condition must be of the form

$$
D(\mathbf{x}+\mathbf{y})=\left(\Lambda^{\prime}(\mathbf{x}) \Lambda(0)^{\dagger}\right)^{2}(D(\mathbf{y})-D(0))+D(\mathbf{x}) .
$$

Proof. To check the first order condition, it is sufficient to check it only for the unitary generators of $\mathcal{A}_{\theta}$ :

$$
\left[\left[D, U_{\mathbf{x}}\right], U_{\mathbf{y}}^{\mathrm{o}}\right]=D U_{\mathbf{x}} U_{\mathbf{y}}^{0}-U_{\mathbf{x}} D U_{\mathbf{y}}^{\mathrm{o}}-U_{\mathbf{y}}^{\mathrm{o}} D U_{\mathbf{x}}+U_{\mathbf{y}}^{\mathrm{o}} U_{\mathbf{x}} D=0
$$

for all $\mathbf{x}, \mathbf{y} \in \mathbb{Z}^{n}$. Using Lemma 2, we write out the first order condition:

$$
\begin{aligned}
& D U_{\mathbf{x}} U_{\mathbf{y}}^{0} e_{\boldsymbol{\mu}, j}=a(\mathbf{x}, \mathbf{y}, \boldsymbol{\mu}) D(\mathbf{x}+\mathbf{y}+\boldsymbol{\mu}) \Lambda^{\prime}(\mathbf{y}) \Lambda^{\prime}(0)^{\dagger} e_{\boldsymbol{\mu}+\mathbf{x}+\mathbf{y}, j} \\
& U_{\mathbf{x}} D U_{\mathbf{y}}^{0} e_{\boldsymbol{\mu}, j}=a(\mathbf{x}, \mathbf{y}, \boldsymbol{\mu}) D(\mathbf{y}+\boldsymbol{\mu}) \Lambda^{\prime}(\mathbf{y}) \Lambda^{\prime}(0)^{\dagger} e_{\boldsymbol{\mu}+\mathbf{x}+\mathbf{y}, j} \\
& U_{\mathbf{y}}^{0} D U_{\mathbf{x}} e_{\boldsymbol{\mu}, j}=a(\mathbf{x}, \mathbf{y}, \boldsymbol{\mu}) \Lambda^{\prime}(\mathbf{y}) \Lambda^{\prime}(0)^{\dagger} D(\mathbf{x}+\boldsymbol{\mu}) e_{\boldsymbol{\mu}+\mathbf{x}+\mathbf{y}, j} \\
& U_{\mathbf{y}}^{0} U_{\mathbf{x}} D e_{\boldsymbol{\mu}, j}=a(\mathbf{x}, \mathbf{y}, \boldsymbol{\mu}) \Lambda^{\prime}(\mathbf{y}) \Lambda^{\prime}(0)^{\dagger} D(\boldsymbol{\mu}) e_{\boldsymbol{\mu}+\mathbf{x}+\mathbf{y}, j} .
\end{aligned}
$$

Here $a(\mathbf{x}, \mathbf{y}, \boldsymbol{\mu})$ is the common factor

$$
a(\mathbf{x}, \mathbf{y}, \boldsymbol{\mu})=e\left(\mathbf{x} \cdot \mathbf{A} \boldsymbol{\mu}+\boldsymbol{\mu} \cdot \mathbf{A y}+\mathbf{x} \cdot \mathbf{A y}+\frac{1}{2}(\mathbf{x} \cdot \mathbf{A x}+\mathbf{y} \cdot \mathbf{A y})\right) .
$$

This gives the relation

$$
(D(\mathbf{x}+\mathbf{y}+\boldsymbol{\mu})-D(\mathbf{y}+\boldsymbol{\mu})) \Lambda^{\prime}(\mathbf{y}) \Lambda^{\prime}(0)^{\dagger}=\Lambda^{\prime}(\mathbf{y}) \Lambda^{\prime}(0)^{\dagger}(D(\mathbf{x}+\boldsymbol{\mu})-D(\boldsymbol{\mu})) .
$$

Since $D$ is self-adjoint and $\Lambda^{\prime}(\mathbf{x})$ unitary, we can rewrite this as

$$
(D(\mathbf{x}+\mathbf{y}+\boldsymbol{\mu})-D(\mathbf{y}+\boldsymbol{\mu}))=\left(\Lambda^{\prime}(\mathbf{y}) \Lambda^{\prime}(0)^{\dagger}\right)^{2}(D(\mathbf{x}+\boldsymbol{\mu})-D(\boldsymbol{\mu})) .
$$

For $\mathbf{y}=\mathbf{x}$ and $\boldsymbol{\mu}=0$, the solution to the defining equation (15) is

$$
D(\mathbf{x})=\sum_{i}\left(\boldsymbol{\tau}_{i} \cdot \mathbf{x}\right) A_{i}+\left(\Lambda^{\prime}(\mathbf{x}) \Lambda(0)^{\dagger}\right)^{2} B+C,
$$

where the $A_{i}, B$ and $C$ are bounded operators such that $C e_{\boldsymbol{\mu}, j}=\sum_{k} c_{j k} e_{\boldsymbol{\mu}, k}$ and similarly for $B$ and $A_{i}$, and $\operatorname{ker}\left(\sum_{i} \boldsymbol{\tau}_{i} \cdot \mathbf{x} A_{i}\right)$ contains at least the $\mathbf{x} \in \mathbb{Z}^{n}$ such that $\left(\Lambda^{\prime}(\mathbf{x}) \Lambda(0)^{\dagger}\right)^{2} \neq \mathrm{Id}$. This is the unique solution, since we see from equation (15) that $D$ is fully determined after we choose suitable $D\left(e_{i}\right)$ for $1 \leq i \leq n$.

If $\left(\Lambda^{\prime}(\mathbf{x}) \Lambda(0)^{\dagger}\right)^{2}=$ Id, this gives a linear Dirac operator, familiar from commutative geometry. However, at first glance it seems that there might be other spin structures, not corresponding to commutative spin geometries. These other candidate geometries, where $\left(\Lambda^{\prime}(\mathbf{x}) \Lambda(0)^{\dagger}\right)^{2} \neq \mathrm{Id}$, will however drop out because they are incompatible with the compact resolvent condition on $D$. 
Lemma 4. Only if $\left(\Lambda^{\prime}(\mathbf{x}) \Lambda(0)^{\dagger}\right)^{2}=\mathrm{Id}$ can the equivariant Dirac operator $D$ have a compact resolvent. Hence $D$ is of the form

$$
D e_{\mu, j}=\left(\sum_{i}\left(\boldsymbol{\tau}_{i} \cdot \boldsymbol{\mu}\right) A_{i}+C\right) e_{\boldsymbol{\mu}, j} .
$$

As a corollary, we have that $J$ is of the form

$$
J e_{\boldsymbol{\mu}, j}=e(\boldsymbol{\mu} \cdot \mathbf{A} \boldsymbol{\mu}) \Lambda e_{-\boldsymbol{\mu}, j},
$$

with $\Lambda$ a constant isometry such that $\Lambda^{2}=\epsilon_{J}$ Id.

Proof. By [29], Theorem XIII.64, we see that an unbounded self-adjoint operator $D$ bounded away from 0 has a compact resolvent if and only if the set

$$
F_{b}=\{\psi \in \operatorname{Dom}(D) \mid\|\psi\| \leq 1,\|D \psi\| \leq b\}
$$

is compact for all $b \in \mathbb{R}$. However, if $\left(\Lambda^{\prime}(\mathbf{x}) \Lambda(0)^{\dagger}\right)^{2} \neq$ Id for some direction $\mathbf{x}$, we know that $\mathbf{x} \in \operatorname{ker}\left(\sum_{i} \boldsymbol{\tau}_{i} \cdot \mathbf{x} A_{i}\right)$, and then it follows from (16) that the norm of $D e_{\lambda \mathbf{x}}$ is bounded by $B+C$ for all $\lambda \in \mathbb{Z}$. When we take $b>\|B+C\|, F_{b}$ contains at least $e_{\lambda \mathbf{x}}$ for all $\lambda \in \mathbb{Z} \neq 0$, so $F_{b}$ cannot be compact.

\section{Grading and Hochschild homology}

In this section we investigate what extra conditions on the spectral triple of the noncommutative torus come from the grading operator and Hochschild conditions, Conditions 2 and 6. We find that the parameters $\tau^{i}$ introduced in Section 6 must be linearly independent vectors spanning $\mathbb{R}^{n}$.

We start by investigating the Hochschild cycle condition, which states that there is a Hochschild cocycle $c \in Z_{n}\left(\mathcal{A}, \mathcal{A} \otimes \mathcal{A}^{\circ}\right)$ whose representative on $\mathscr{H}$ is $\Gamma$ when $n$ is even, or Id if $n$ is odd. The $\Gamma$ operator is an isometry, with eigenvalues 1 and -1 , and so by Condition 2 and the diagonal action of the algebra on the Hilbert space, we see that

$$
\Gamma=\left(\begin{array}{cc}
\Gamma^{+} & 0 \\
0 & \Gamma^{-}
\end{array}\right)
$$

where $\Gamma^{+}$and $\Gamma^{-}$are unitary self-adjoint operators which have only eigenvalues +1 and -1 respectively. Using a unitary transformation, we can assume these operators to be diagonal, thus

$$
\Gamma=\left(\begin{array}{cc}
\mathrm{Id}_{+} & 0 \\
0 & -\mathrm{Id}_{-}
\end{array}\right),
$$

where $\mathrm{Id}_{+}$and $\mathrm{Id}_{-}$are the identity operators on the positive and negative eigenspaces of $\Gamma$. 
An obvious candidate for the Hochschild cycle in $Z_{n}\left(\mathcal{A}, \mathcal{A} \otimes \mathcal{A}^{\circ}\right)$ is the straightforward generalization of the unique such cycle for the noncommutative 2-torus [28], p. 324, which is

$$
c_{2}=U_{(1,0)}^{*} U_{(0,1)}^{*} \otimes U_{(0,1)} \otimes U_{(1,0)}-U_{(0,1)}^{*} U_{(1,0)}^{*} \otimes U_{(1,0)} \otimes U_{(0,1)} .
$$

A candidate generator of the $n$-th Hochschild homology of the noncommutative $n$ torus is given by

$$
c_{n}=\sum_{\sigma \in S_{n}}\left(\operatorname{sign}(\sigma)\left(\prod_{i=1}^{n} U_{e_{\sigma(i)}}\right)^{*} \bigotimes_{i=1}^{n}\left(U_{e_{\sigma(i)}}\right)\right),
$$

with $e_{i}$ an orthonormal basis of $\mathbb{Z}^{n}$. It is known by [22], Lemma 12.15, that this a Hochschild cycle. Due to [38], Theorem 1.1, the $n$-th Hochschild homology of the $n$-torus is 1-dimensional. Together with Lemmas 5 and 6 below, this means that (18) generates the $n$-th Hochschild homology.

Lemma 5. For the noncommutative n-torus, only nontrivial cycles can be mapped to $\Gamma$ when $n$ is even, and to $\mathrm{Id}$ when $n$ is odd, by the map $\pi_{D}$.

Proof. Since the Hochschild cycle consists of polynomial expressions, it is enough to prove the result for individual homogeneous polynomials because any cycle can be written as the sum of homogeneous polynomials. Define

$$
c^{\prime}=U_{\mathbf{x}^{0}} \otimes U_{\mathbf{y}}^{\mathrm{o}} \otimes U_{\mathbf{x}^{1}} \otimes \cdots \otimes U_{\mathbf{x}^{n}} \in Z_{n+1}\left(\mathcal{A}, \mathcal{A} \otimes \mathcal{A}^{\mathrm{o}}\right),
$$

with $\mathbf{x}^{i}, \mathbf{y} \in \mathbb{Z}^{n}$. As in [37], Chapter 3.5, we see that

$$
\pi_{D}\left(b c^{\prime}\right)=(-1)^{n}\left[\pi^{\mathrm{o}}\left(U_{\mathbf{y}}\right) \pi\left(U_{\mathbf{x}^{0}}\right)\left[D, \pi\left(U_{\mathbf{x}^{1}}\right)\right] \ldots\left[D, \pi\left(U_{\mathbf{x}^{n}}\right)\right], \pi\left(U_{\mathbf{x}^{n+1}}\right)\right] .
$$

Since $\left[D, U_{\mathbf{x}}\right]=C_{\mathbf{x}} U_{\mathbf{x}}$ with $C_{\mathbf{x}}$ some operator depending on $\mathbf{x}, \pi_{D}\left(b c^{\prime}\right)$ is proportional to

$$
C_{\mathbf{x}^{1}, \ldots, \mathbf{x}^{n}} \pi\left(U_{\mathbf{x}^{0}}\right) \pi^{\mathrm{o}}\left(U_{\mathbf{y}}\right) \pi\left(U_{\mathbf{x}^{1}}\right) \ldots \pi\left(U_{\mathbf{x}^{n}}\right) \pi\left(U_{\mathbf{x}^{n+1}}\right) .
$$

When $n$ is even, $\Gamma$ maps $e_{\boldsymbol{\mu}, j}$ to $\pm e_{\boldsymbol{\mu}, j}$, the total sum $\mathbf{y}+\sum_{i=0}^{n+1} \mathbf{x}^{i}$ must be 0 . If $\mathcal{A}\left(\mathbb{T}_{\theta}^{n}\right)$ and $\mathcal{A}\left(\mathbb{\mathbb { T }}_{\theta}^{n}\right)^{\mathrm{o}}$ have a trivial intersection, $U_{\mathbf{y}}^{\mathrm{o}}$ must have total degree 0 . The total $\sum_{i=0}^{n+1} \mathbf{x}^{i}$ is 0 , and since $U_{\mathbf{x}}$ and $U_{-\mathbf{x}}$ commute, the commutator (19) vanishes. When $n$ is odd, the argument goes the same.

If $\mathcal{A}\left(\mathbb{T}_{\theta}^{n}\right)$ and $\mathcal{A}\left(\mathbb{\mathbb { T }}_{\theta}^{n}\right)^{\mathrm{o}}$ have nontrivial intersection, there are $\mathbf{y}$ for which $U_{\mathbf{x}} U_{\mathbf{y}}=$ $U_{\mathbf{y}} U_{\mathbf{x}}$ for all $\mathbf{x} \in \mathbb{Z}^{n}$. In that case, by the same arguments as for the trivial intersection case above, the $U_{\mathbf{y}}$ must lie entirely within the intersection of $\mathcal{A}\left(\mathbb{T}_{\theta}^{n}\right)$ and $\mathcal{A}\left(\mathbb{T}_{\theta}^{n}\right)^{\mathrm{o}}$, and since $\mathbf{x}^{n+1}=-\mathbf{y}-\sum_{i=0}^{n} \mathbf{x}^{i}$, the commutator (19) vanishes:

$$
\begin{aligned}
{\left[U_{\mathbf{y}}^{\mathrm{o}} U_{\mathbf{x}^{1}} \ldots U_{\mathbf{x}^{n}}, U_{\mathbf{x}^{n+1}}\right] } & =U_{\mathbf{y}}^{\mathrm{o}}\left[U_{\mathbf{x}^{1}} \ldots U_{\mathbf{x}^{n}}, U_{\mathbf{x}^{n+1}}\right] \\
& =U_{\mathbf{y}}^{\mathrm{o}}\left(1-e\left(\mathbf{x}^{n+1} \cdot \theta \sum_{i}^{n} \mathbf{x}^{i}\right)\right) \\
& =U_{\mathbf{y}}^{\mathrm{o}}\left(1-e\left(\mathbf{x}^{n+1} \cdot \theta\left(-\mathbf{y}-\mathbf{x}^{n+1}\right)\right)\right)=0 .
\end{aligned}
$$

Since $\mathbf{x}^{n+1} \cdot \theta \mathbf{x}^{n+1}=0$ and $U_{\mathbf{y}}$ commutes with $U_{\mathbf{x}^{n+1}}$. 
Lemma 6. The Dirac operator given in Lemma 4 satisfies the Hochschild condition only if the vectors $\boldsymbol{\tau}^{i}$ are linearly independent. The Dirac operator is

$$
D=\sum_{i}\left(\boldsymbol{\tau}^{i} \cdot \boldsymbol{\delta}\right) A_{i}+C,
$$

where the $A_{i}$ are bounded operators such that

$$
\sum_{\sigma \in S_{n}} \operatorname{sign}(\sigma) \prod_{i} A_{\sigma(i)}=\operatorname{det}\left(\boldsymbol{\tau}^{1} \boldsymbol{\tau}^{2} \ldots \boldsymbol{\tau}^{n}\right) \Gamma
$$

when $n$ is even, and $\operatorname{det}\left(\boldsymbol{\tau}^{1} \boldsymbol{\tau}^{2} \ldots \boldsymbol{\tau}^{n}\right) \operatorname{Id}$ when $n$ is odd.

Proof. In order to deduce the representative of the Hochschild cycle (18) on the Hilbert space $\mathscr{H}$, we calculate

$$
\begin{aligned}
{\left[D, U_{\mathbf{x}}\right] e_{\mu, j} } & =D e\left(\mathbf{x} \cdot \mathbf{A} \boldsymbol{\mu}+\frac{1}{2} \mathbf{x} \cdot \mathbf{A x}\right) e_{\boldsymbol{\mu}+\mathbf{x}, j}-U_{\mathbf{x}}\left(\sum_{k} \boldsymbol{\tau}_{k} \cdot \boldsymbol{\mu} A_{k} e_{\boldsymbol{\mu}, j}\right) \\
& =e\left(\mathbf{x} \cdot \mathbf{A} \boldsymbol{\mu}+\frac{1}{2} \mathbf{x} \cdot \mathbf{A x}\right)\left(\sum_{k} \boldsymbol{\tau}_{k} \cdot \mathbf{x} A_{k} e_{\boldsymbol{\mu}+\mathbf{x}, j}\right) .
\end{aligned}
$$

We suppress the $e\left(\mathbf{x} \cdot \mathbf{A} \boldsymbol{\mu}+\frac{1}{2} \mathbf{x} \cdot \mathbf{A x}\right)$ factors, since these are canceled from the left by the $U_{e_{i}}^{*}$ factors, and expand (18):

$$
\begin{aligned}
\pi_{D}(\boldsymbol{c}) & =\pi_{D}\left(\sum_{\sigma \in S_{n}}\left(\operatorname{sign}(\sigma)\left(\prod_{i=1}^{n} U_{e_{\sigma(i)}}\right)^{*} \bigotimes_{i=1}^{n}\left(U_{e_{\sigma(i)}}\right)\right)\right) \\
& =\sum_{\sigma \in S_{n}}\left(\operatorname{sign}(\sigma)\left(\prod_{i=1}^{n} U_{e_{\sigma(i)}}\right)^{*} \prod_{i=1}^{n}\left[D, U_{e_{\sigma(i)}}\right]\right) \\
& =\sum_{\sigma \in S_{n}}\left(\operatorname{sign}(\sigma) \sum_{k} \prod_{i=1}^{n} \tau^{k} \cdot e_{\sigma(i)} A_{k}\right) .
\end{aligned}
$$

This expression should be some constant times $\Gamma$ or Id, depending on the dimension. Due to [10], Proposition 4.2, we can write this as

$$
\pi_{D}(\boldsymbol{c})=\operatorname{det}\left(\boldsymbol{\tau}_{1} \boldsymbol{\tau}_{2} \ldots \boldsymbol{\tau}_{n}\right) \sum_{\sigma \in S_{n}} \operatorname{sign}(\sigma) \prod_{i} A_{\sigma(i)},
$$

where we view $\left(\boldsymbol{\tau}_{1} \boldsymbol{\tau}_{2} \ldots \boldsymbol{\tau}_{n}\right)$ an $(n \times n)$-matrix with $\boldsymbol{\tau}_{i}$ as the columns, from which it is immediately clear that the vectors must be linearly independent.

\section{Dimension, finiteness and regularity}

Here we establish, using the conditions of dimension, regularity and finiteness (Conditions 4, 7 and 8), that the real spectral triple must be an isospectral deformation of a spin structure on a noncommutative torus. The result follows from Connes' spin manifold theorem. In the course of proving this, we find a proof for an elementary fact about Hermitian matrices generating a Clifford algebra, for which we do not know an elementary proof. 
Lemma 7. If the $\boldsymbol{\tau}_{i}$ span $\mathbb{R}^{n}$, for arbitrary $a_{i} \in \mathbb{R}$ and all $\varepsilon>0$, there is a $t \in \mathbb{R}$ and a finite set of $\mu_{j} \in \widetilde{\mathbb{Z}}^{n}$, with $\widetilde{\mathbb{Z}}^{n}$ a shifted lattice as in Section 5, such that

$$
\sum_{i}\left|t a_{i}-\boldsymbol{\tau}_{i} \cdot \sum_{j} \boldsymbol{\mu}_{j}\right|<\varepsilon
$$

Proof. Every vector $\mathbf{p} \in \mathbb{Z}^{n}$ can be written as a sum of at most two vectors $\boldsymbol{\mu}_{j} \in$ $\widetilde{\mathbb{Z}}^{n}$. By Dirichlet's theorem of simultaneous Diophantine approximation [33], Theorem II.1B, for all $N>1$ and $\left(a_{i}^{\prime}\right) \in \mathbb{R}^{n}$ we can find integers $q, p_{1}, \ldots, p_{n}$ with $q<N$ such that

$$
\left|q a_{i}^{\prime}-p_{i}\right|<N^{-1 / n}
$$

for $1 \leq i \leq n$ and $a_{i} \in \mathbb{R}$.

Since the $\tau_{i}$ span $\mathbb{R}^{n}$, there is a transformation $R \in \operatorname{GL}(\mathbb{R}, n)$ depending only on the $\boldsymbol{\tau}_{i}$ such that $(R \mathbf{p})_{i}=\boldsymbol{\tau}_{i} \cdot \mathbf{p}$. Set $a_{i}^{\prime}=\left(R^{-1} \mathbf{a}\right)_{i}$. Call the eigenvalue of $R$ with the maximal absolute value $\tau_{\max }$, then clearly we have

$$
\left|q a_{i}-\boldsymbol{\tau}_{i} \cdot \sum_{j} \boldsymbol{\mu}_{j}\right|=\left|q\left(R \mathbf{a}^{\prime}\right)_{i}-(R \mathbf{p})_{i}\right| \leq\left|\tau_{\max }\right|\left|q a_{i}^{\prime}-p_{i}\right|<\left|\tau_{\max }\right| N^{-1 / n} .
$$

Choosing $N$ such that $n\left|\tau_{\max }\right| N^{-1 / n}<\varepsilon$, we get the desired result.

Lemma 8. In order for D to be satisfy the compact resolvent condition of Condition 1 and the dimension condition of Condition 4,

$$
\sum_{i} x_{i} A_{i}
$$

needs to be invertible for all $x_{i} \in \mathbb{R}$ except when all $x_{i}$ vanish. In particular, all $A_{i}$ should be invertible operators.

Proof. If $\sum_{i} x_{i} A_{i}$ is not invertible at a point where $x_{i}=\boldsymbol{\tau}_{i} \cdot \boldsymbol{\mu}$ for all $i$, then clearly this is also the case for $\mu^{\prime}=\lambda \mu$ with $\lambda \in \mathbb{Z}$. Thus the kernel cannot be finite dimensional in this case.

If $\sum_{i} x_{i} A_{i}$ is not invertible for some non-trivial $x_{i}$, but $x_{i} \neq \boldsymbol{\tau}_{i} \cdot \boldsymbol{\mu}$ for all $\boldsymbol{\mu} \in \mathbb{Z}^{n}$ we have the following.

Since the $\boldsymbol{\tau}_{i}$ span $\mathbb{R}^{n}$ by Lemma 6 , by Lemma 7 we have for every $\varepsilon>0$ a set of vectors $\boldsymbol{\mu}_{j} \in \mathbb{Z}^{n}$ such that $\sum_{i}\left|\sum_{j} \boldsymbol{\tau}_{i} \cdot \boldsymbol{\mu}_{j}-x_{i}\right|<\varepsilon$. Take an element $\mathbf{y}$ in the kernel of $\sum_{i} x_{i} A_{i}$. Then $\left\|\left(\sum_{i, j} \boldsymbol{\tau}_{i} \cdot \boldsymbol{\mu}_{j} A_{i}\right) \cdot \mathbf{y}\right\|<\varepsilon\|\mathbf{y}\|$. Hence there is at least one eigenvalue of $\sum_{i, j} \boldsymbol{\tau}_{i} \cdot \mu_{j} A_{i}$ smaller or equal to $\varepsilon$. But this means that the spectrum of $|D|^{-1}$ is unbounded, hence $D^{-1}$ cannot be compact.

We have so far assumed nothing about the size of the Hilbert space $\mathscr{H}$ compared to the basic irreducible representation of the algebra, $\mathscr{H}_{0}$ as defined in equation (4). By construction, $\mathscr{H}$ is a left $\mathcal{A}\left(\mathbb{T}_{\theta}^{n}\right)$-module. According to Condition 8 , a certain submodule $\mathscr{H}^{\infty}$ of $\mathscr{H}$ should be a finitely generated projective left $\mathcal{A}\left(\mathbb{T}_{\theta}^{n}\right)$-module. This has the following consequence: 
Lemma 9. The spectral triple $(\mathcal{A}, \mathcal{H}, D)$ only satisfies the finiteness condition of Condition 8 if the Hilbert space $\mathscr{H}$ is a finite direct sum of copies of $\mathcal{H}_{0}$. If we assume the algebra $\mathcal{A}$ to be closed under the collection of seminorms $\left\|\delta^{k}(\cdot)\right\|$ defined in Condition 7, it is given by

$$
\mathcal{A}\left(\mathbb{T}_{\theta}^{n}\right)=\left\{\sum_{\mathbf{x} \in \mathbb{Z}^{n}} a(\mathbf{x}) U_{\mathbf{x}} \mid a(\mathbf{x}) \in S\left(\mathbb{Z}^{n}\right)\right\},
$$

where $S\left(\mathbb{Z}^{n}\right)$ is the set of Schwartz functions over $\mathbb{Z}^{n}$ :

$$
\delta\left(\mathbb{Z}^{n}\right)=\left\{a:\left.\mathbb{Z}^{n} \rightarrow \mathbb{C}\left|\sup _{\mathbf{x} \in \mathbb{Z}^{n}}\left(1+\|\mathbf{x}\|^{2}\right)^{k}\right| a(\mathbf{x})\right|^{2}<\infty \text { for all } k \in \mathbb{N}\right\} .
$$

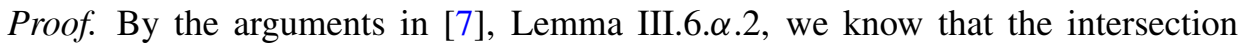
of the domain of the $\delta^{k}$ is stable under the holomorphic functional calculus. The collection of seminorms $\left\|\delta^{k}(\cdot)\right\|$ is equivalent to the collection of seminorms $q_{k}=$ $\sup _{\mathbf{x} \in \widetilde{\mathbb{Z}}^{n}}\left(1+|\mathbf{x}|^{2}\right)^{k}|a(\mathbf{x})|$ because of the following argument.

We can write $\left\|\delta^{k}(a)\right\|^{2}=\sum_{i} \sum_{\mathbf{x} \in \mathbb{Z}^{n}}\left\|A_{i}\right\|^{2 k}\left\|\boldsymbol{\tau}_{i} \cdot \mathbf{x}\right\|^{2 k}|a(x)|^{2}$. Because of Lemmas 6 and 8, we know that $\sum_{i}\left\|A_{i}\right\|^{2 k}\left\|\boldsymbol{\tau}_{i} \cdot \mathbf{x}\right\|^{2 k}$ can be bounded between $c_{1, k}\|\mathbf{x}\|^{2 k}$ and $c_{2, k}\|\mathbf{x}\|^{2 k}$ for constants $c_{1, k}, c_{2, k}>0$. Thus the family of seminorms $\left\|\delta^{k}(a)\right\|$ is equivalent to the family of seminorms $q_{k}^{\prime}(a)=\sum_{\mathbf{x} \in \mathbb{Z}^{n}}\|\mathbf{x}\|^{2 k}|a(\mathbf{x})|^{2}$. Now if an element $a$ of the $\mathrm{C}^{*}$-algebra $A$ has a finite norm in all $q_{k}^{\prime}$, then clearly $\sup _{\mathbf{x} \in \mathbb{Z}^{n}}\left(1+\|\mathbf{x}\|^{2}\right)^{k}|a(\mathbf{x})|^{2}<\infty$ for all $k \in \mathbb{N}$. Conversely, if $a \in \mathcal{A}\left(\mathbb{T}_{\theta}^{n}\right)$, we can write

$$
q_{k}^{\prime}(a)=\sum_{\mathbf{x} \in \mathbb{Z}^{n}}\|\mathbf{x}\|^{2 k}|a(\mathbf{x})|^{2}=\sum_{\mathbf{x} \in \mathbb{Z}^{n}}\left(1+\|\mathbf{x}\|^{2}\right)^{p}\left(1+\|\mathbf{x}\|^{2}\right)^{-p}\|\mathbf{x}\|^{2 l}|a(\mathbf{x})|^{2},
$$

and this last sum converges if $p$ is big enough since $\left(1+\|\mathbf{x}\|^{2}\right)^{p}|a(\mathbf{x})|^{2}$ is by assumption less than some finite constant $c_{p}$ and $\sum_{\mathbf{x} \in \mathbb{Z}^{n}} c_{p}\|\mathbf{x}\|^{2 k}(1+\|\mathbf{x}\|)^{-p}$ converges when $p>n / 2+k$.

By Condition 8 , we have that $\mathscr{H}^{\infty}$ is a finitely generated projective left $\mathcal{A}\left(\mathbb{T}_{\theta}^{n}\right)$ module. We already knew that $\mathscr{H}$ must be a direct sum of copies of $\mathscr{H}_{0}$ due to the discussion following (10). The finiteness condition then ensures that the sum must be finite. All conditions stated in Conditions 7 and 8 are then easily seen to be fulfilled.

Remark 1. The choice of smooth structure is not unique even in the commutative case, given just the algebra $\mathrm{C}^{*}$-algebra $A$ of the noncommutative torus. See for example [23]. However, given the equivariance condition of the Dirac operator and the assumption that the smooth algebra consists of all elements such that $\left\|\delta^{k}(\cdot)\right\|<\infty$ for all $k \in \mathbb{N}$, the algebra is uniquely determined by the above argument.

The number of generators of $\mathscr{H}$ in terms of $\mathscr{H}_{0}$ is still undetermined, but a lower bound is given by [2], Theorem 1. This theorem states that for complex Hermitian $k \times k$ matrices, with $k=(2 a+1) 2^{b}$, there exists at most $2 b+1$ matrices satisfying the 
non-invertibility property of Lemma 8 . So in order to have at least $n$ such matrices, $k$ should be at least $2^{\lfloor n / 2\rfloor}$. Hermitian matrices generating an irreducible representation of a Clifford algebra $\mathrm{Cl}_{n, 0}$ are an example of a set of matrices attaining this lower bound.

Remark 2. It does not follow from Lemma 8 that the algebra generated by the matrices $A_{i}$ is a Clifford algebra. What remains to be shown is that $\left(\sum_{i} x_{i} A_{i}\right)^{2}$ lies in the center of the bounded operators on $\mathscr{H}$ for all $\mathbf{x} \in \mathbb{R}^{n}$. The condition of Lemma 8 alone is not enough to show this.

Consider a set of self-adjoint matrices $\left\{B_{i}\right\}$ generating a Clifford algebra. These satisfy the invertibility condition of Lemma 8 , and the Hochschild condition (20). Since they are self-adjoint, we can diagonalize $B_{1}$ by $B_{1}=U \Lambda U^{\dagger}$, with $\Lambda$ a diagonal matrix with real elements. If we rescale the elements of $\Lambda$ each by a different nonzero amount to $\Lambda^{\prime}$, the set $\left\{U \Lambda^{\prime} U^{\dagger}\right\} \cup\left\{B_{i}\right\}_{i \geq 2}$ still has the invertibility property, but not necessarily the Hochschild property, as a calculation for any case $n \geq 4$ will show. If $n=2,3$, the invertibility property does imply the Hochschild property however.

However, a weaker form of Connes' reconstruction theorem [10], Theorem 11.5, implies the following result:

Lemma 10. In order for the candidate structure $\left(\mathcal{A}\left(\mathbb{T}_{\theta}^{n}\right), \mathcal{H}, D, J\right)$ to satisfy both the Hochschild condition of Condition 6 and the dimension condition of Condition 4, the matrices $A_{i}$ of Lemma 4 must generate a Clifford algebra.

Proof. If we look at our conditions on $\mathcal{A}\left(\mathbb{T}_{\theta}^{n}\right), \mathscr{H}, D$ and $J$, we see that none of them depend on the antisymmetric matrix $\theta$. Also, the action of the Dirac operator on the Hilbert space is independent of $\theta$. This means that we can just set $\theta=0$, where we have the real spectral triple $\left(\mathcal{A}_{0}, \mathscr{H}, D, J\right)$ of smooth functions on the $n$-torus. Due to the results of Connes' spin manifold theorem (see for example [22], Lemma 11.6, [10], Remark 5.12), this implies that the $A_{i}$ generate a Clifford algebra.

Because the size of the maximal set of matrices which satisfy the invertibility condition of Lemma 8 is odd, due to [2], Theorem 1, we have as a corollary:

Corollary 1. A set of $\left(2^{b} \times 2^{b}\right)$ Hermitian matrices $\left\{A_{i}\right\}_{i=1}^{n}$, where $n=2 b+1$, such that the equation

$$
\operatorname{det}\left(\sum_{i} x_{i} A_{i}\right)=0
$$

only has the zero solution $\left(x_{i}=0\right)_{i=1}^{n}$ in $\mathbb{R}^{n}$ generate a Clifford algebra if and only if

$$
\sum_{\sigma \in S_{n}} \operatorname{sign}(\sigma) \prod_{i}^{n} A_{\sigma(i)}=\lambda \operatorname{Id}_{k}
$$

for some nonzero $\lambda \in \mathbb{R}$. 
If we assume the spectral triple to be irreducible, we need that the matrices generate an irreducible representation of the Clifford algebra. This restricts the size of the matrices to be exactly $2^{\lfloor n / 2\rfloor}$.

From the results in Sections 4, 5 and 6 it now follows that the remaining conditions, the Poincare duality of Condition 9 and the Hermitian pairing of Condition 8 are also satisfied, since they are satisfied by isospectral deformations.

This completes the proof of Theorem A:

Theorem A. All irreducible real spectral triples with an equivariant $n$-torus actions are isospectral deformations of spin structures on an n-torus.

\section{Description of the real spectral triples}

Finally, we show that given a Dirac operator $D$ that satisfies all conditions so far, the reality operator $J$ is uniquely determined, and list all the ingredients that constitute all real spectral triples of the noncommutative $n$-torus. Also, we show in some lowdimensional cases what freedom still exists in the definition of the Dirac operator.

Recall that the Clifford algebra $\mathrm{Cl}_{n, 0}$ is the algebra over $\mathbb{R}$ generated by $\mathbb{R}^{n}, 1$ and a positive definite quadratic form $q$ subject to the relation $v \cdot v=-q(v)$ Id. The Clifford group is defined as the group generated by the image of an orthonormal basis of $\mathbb{R}^{n}$ together with -1 .

Lemma 11. If $D$ is given by $\sum_{j}\left(\boldsymbol{\tau}^{j} \cdot \boldsymbol{\delta}\right) A_{j}$ with $A_{j}$ representatives of the Clifford group of $\mathrm{Cl}_{n, 0}$ there is a unique $J$ operator for each $n$, up to multiplication with a complex number of norm 1 .

Proof. If $d=1,2,3$ or 4 this can be done for example by calculations, see Remark 3 below. We proceed by induction. First we prove existence. Recall that there are isomorphisms $\mathrm{Cl}_{n+2,0} \simeq \mathrm{Cl}_{0, n} \otimes \mathrm{Cl}_{2,0}$ and $\mathrm{Cl}_{0, n+2} \simeq \mathrm{Cl}_{n, 0} \otimes \mathrm{Cl}_{0,2}$ by [26], Theorem I.4.1. Let $d>4$, and assume that the lemma has been proven for $n-4$. The operator $J_{n}=J_{n-4} \otimes J_{4}$ acting on $\mathrm{Cl}_{n, 0} \cong \mathrm{Cl}_{n-4,0} \otimes \mathrm{Cl}_{4,0}$ has precisely the right commutation relations, except for $n \equiv 1 \bmod 4$, as can easily be calculated by looking at Table 1 and taking into account the periodicity mod 4 of the table, except for the first row, where we use $J_{4}^{2}=-1$. In case $n \equiv 1 \bmod 4$, we can achieve the same by setting $J_{n}=J_{n-4} \otimes \Gamma_{4} J_{4}$.

Now we prove the uniqueness. Write $\gamma_{n}^{i}$ for the representation of the $i$-th basis vector of $\mathbb{R}^{n}$ in $\mathrm{Cl}_{n, 0}$. An explicit isomorphism $\mathrm{Cl}_{n, 0} \equiv \mathrm{Cl}_{n-4,0} \otimes \mathrm{Cl}_{4,0}$ can be chosen, for example,

$$
\gamma_{n}^{i}=\operatorname{Id}_{n-4} \otimes \gamma_{4}^{i} \text { for } i \leq 4, \quad \gamma_{n}^{i}=\gamma_{n-4}^{i-4} \otimes \gamma_{4}^{1} \gamma_{4}^{2} \gamma_{4}^{3} \gamma_{4}^{4} \text { for } i>4 .
$$

The operators $\gamma_{n}^{1} \gamma_{n}^{2}$ and $\gamma_{n}^{3} \gamma_{n}^{4}$ commute with $\gamma_{n}^{i}$ for $i>4$ and anticommute with $\gamma_{n}^{1}$, $\gamma_{n}^{2}$ and $\gamma_{n}^{3}, \gamma_{n}^{4}$, respectively. They square to -1 , so the operator

$$
P^{+}=: \frac{1}{4}\left(1+i \gamma_{n}^{1} \gamma_{n}^{2}\right)\left(1+i \gamma_{n}^{3} \gamma_{n}^{4}\right)
$$


is a projection, which commutes with $\gamma_{n}^{i}$ for $i>4$. The projection $P^{+}$does not commute with $J_{n}$ but does commute with $\gamma_{2} \gamma_{3} J_{n}$. Also, the projection $P^{+}$projects onto a subspace of dimension $1 / 4$ times the dimension of the irreducible representation of $\mathrm{Cl}_{n, 0}$, and the operator $\gamma_{2} \gamma_{3} J_{n}$ has the same commutation relations with $\gamma_{n}^{i}$ for $i>4$ as $J_{n}$, and since $\left(\gamma_{2} \gamma_{3} J_{n}\right)^{2}=-J_{n}^{2}$, it has the right signs for an $(n-4)$ dimensional $J$ operator. This means that $P^{+}$projects onto a Hilbert space belonging to an $(n-4)$-dimensional spectral triple, where we have a unique $J$ operator by the induction hypothesis. On the complement of the $P^{+}$eigenspace we have the unique $J_{4}$ operator with the right commutation relations with $\gamma_{i}, i \leq 4$.

Stated more elaborately, we have the following result:

Theorem C. The following give all $2^{n}$ irreducible real spectral triples on the smooth noncommutative $n$-torus $\mathcal{A}\left(\mathbb{T}_{\theta}^{n}\right)$ :

- A Hilbert space $\mathscr{H}$ constructed as follows:

$$
\mathscr{H}=\bigoplus_{i}^{2\lfloor n / 2\rfloor} \mathscr{H}^{i}, \quad \mathscr{H}^{i}=\bigoplus_{\mathbf{m} \in \mathbb{Z}^{n}+\boldsymbol{\epsilon}} \mathbb{C},
$$

with $\epsilon=\left(\epsilon_{1}, \ldots, \epsilon_{n}\right) \in \mathbb{R}^{n}$ and $\epsilon_{i} \in\left\{0, \frac{1}{2}\right\}$.

- An involutive algebra $A$ with unitary generators $U_{\mathbf{x}}$ with $\mathbf{x} \in \mathbb{Z}^{n}$,

$$
\mathcal{A}\left(\mathbb{\nabla}_{\theta}^{n}\right)=:\left\{A=\sum_{\mathbf{x}} a(\mathbf{x}) U_{\mathbf{x}} \mid a \in S\left(\mathbb{Z}^{n}\right)\right\},
$$

with $U_{\mathbf{x}}$ acting on a basis vector $e_{\boldsymbol{\mu}, i} \in \mathscr{H}^{i}$ by

$$
U_{\mathbf{x}} e_{\boldsymbol{\mu}, i}=e\left(\frac{1}{2} \mathbf{x} \cdot \mathbf{A} \mathbf{x}+\mathbf{x} \cdot \mathbf{A} \boldsymbol{\mu}\right) e_{\boldsymbol{\mu}+\mathbf{x}, i}
$$

for any matrix $\mathbf{A}$ such that $\mathbf{A}-\mathbf{A}^{t}=\theta$.

- An unbounded, densely defined, self-adjoint first order operator

$$
D=\sum_{j=1}^{n}\left(\boldsymbol{\tau}^{j} \cdot \boldsymbol{\delta}\right) A_{j}+C,
$$

acting on $\mathscr{H}$ with $C$ a bounded self-adjoint operator commuting with the algebra satisfying $J C J^{-1}=\epsilon_{D} C$, and $\Gamma C=-C \Gamma$ if $n$ is even, for $\tau^{j} n$ linearly independent vectors in $\mathbb{R}^{n}, n$ matrices $A_{j}$ of size $2^{\lfloor n / 2\rfloor} \times 2^{\lfloor n / 2\rfloor}$ generating an irreducible representation of the Clifford group $\mathrm{Cl}_{n, 0}$, and $\delta$ the derivations $\delta_{i} e_{\boldsymbol{\mu}}=\mu_{i} e_{\boldsymbol{\mu}}$.

- If $n$ is even, the grading operator $\Gamma$ is given by $\sum_{\sigma \in S_{n}} \operatorname{sign}(\sigma) \prod_{i} A_{\sigma(i)}$, with $A_{j}$ the matrices given above.

- A unique (up to multiplication with a complex number of modulus 1) antilinear isometry $J$ that acts by

$$
J e_{\boldsymbol{\mu}, j}=e(\boldsymbol{\mu} \cdot \mathbf{A} \boldsymbol{\mu}) \Lambda e_{-\boldsymbol{\mu}, j},
$$

with $\Lambda$ a bounded linear operator such that $\Lambda \Lambda^{\dagger}=\operatorname{Id}$ and $D \Lambda=-\epsilon_{D} \Lambda D^{*}$. 
In lower dimensional cases we can explicitly calculate what form $C$ in (21) can take. In [28], Lemma 2.3, Theorem 2.5, it is proven that $C=0$ if $n=2$. For $n=3$ and 4 we have the following:

Proposition 1. If $n=3$ the constant matrix $C$ must have the form $q \mathrm{Id}$, where $q \in \mathbb{R}$ arbitrary. If $n=4$, the matrix $C$ must have the form

$$
\left(\begin{array}{rrrr}
0 & 0 & a & b \\
0 & 0 & -\bar{b} & \bar{a} \\
\bar{a} & -b & 0 & 0 \\
\bar{b} & a & 0 & 0
\end{array}\right),
$$

where $a, b \in \mathbb{C}$.

Proof. If $n=3$ it follows from Theorem C that

$$
J=e(\boldsymbol{\mu} \cdot \mathbf{A} \boldsymbol{\mu}) \sum_{k \in I} a_{j k} e_{-\boldsymbol{\mu}, k},
$$

with $I=\{1,2\}$. We choose a particular form of $D$, given by the particular representation of the Clifford group $\mathrm{Cl}_{3,0}$ known as the Pauli matrices. We see that

$$
J=e(\boldsymbol{\mu} \cdot \mathbf{A} \boldsymbol{\mu})\left(\begin{array}{rr}
0 & -1 \\
1 & 0
\end{array}\right),
$$

just as in the $n=2$ case in [28], Theorem 2.5. Using the appropriate values for $\epsilon_{J}$ and $\epsilon_{D}$ found in Table 1 , we see that the defining equation is $J C J=C$ and by a calculation this shows that $C=q$ Id with $q \in \mathbb{R}$ arbitrary. Similarly for $n=4$, we can just check what the conditions are for $C$ to satisfy the equations $\Gamma D=-D \Gamma$ and $J C=C J$, and this leads to the possibilities given in the proposition. It is trivial to calculate similar conditions for higher dimensions. Due to the increase in the size of matrices, and relaxation of the commutation relation with $\Gamma$ when going from $n=2 k$ to $n=2 k+1$, the number of parameters will increase when $n$ grows.

Remark 3. If we choose a representation for $\mathrm{Cl}_{1,0}, \mathrm{Cl}_{2,0}$ and $\mathrm{Cl}_{0,2}$ all Clifford algebras $\mathrm{Cl}_{n, 0}$ can be constructed by the basic isomorphisms, [26], Theorem I.4.1,

$$
\begin{aligned}
& \mathrm{Cl}_{n, 0} \otimes \mathrm{Cl}_{0,2} \cong \mathrm{Cl}_{0, n+2}, \\
& \mathrm{Cl}_{0, n} \otimes \mathrm{Cl}_{2,0} \cong \mathrm{Cl}_{n+2,0} .
\end{aligned}
$$

We choose a representation

$$
\mathrm{Cl}_{1,0}=1, \quad \mathrm{Cl}_{2,0}=\left(\begin{array}{rr}
0 & i \\
-i & 0
\end{array}\right),\left(\begin{array}{rr}
1 & 0 \\
0 & -1
\end{array}\right), \quad \mathrm{Cl}_{0,2}=\left(\begin{array}{rr}
0 & -1 \\
1 & 0
\end{array}\right),\left(\begin{array}{rr}
i & 0 \\
0 & -i
\end{array}\right) .
$$


The (unique up to multiplication with a complex number of norm 1) matrix part of the $J$ operator can easily be calculated:

$$
J_{2}=J_{3}=\left(\begin{array}{rr}
0 & 1 \\
-1 & 0
\end{array}\right), \quad J_{4}=\left(\begin{array}{rrrr}
0 & 1 & 0 & 0 \\
-1 & 0 & 0 & 0 \\
0 & 0 & 0 & -1 \\
0 & 0 & 1 & 0
\end{array}\right), \quad J_{5}=\left(\begin{array}{rrrr}
0 & 0 & -1 & 0 \\
0 & 0 & 0 & 1 \\
1 & 0 & 0 & 0 \\
0 & -1 & 0 & 0
\end{array}\right)
$$

In this representation, we also see that for all $n$ the matrix component of $J$ has precisely one nonzero element in each column or row.

\section{Unitary equivalences}

After establishing that all real spectral triples on the noncommutative $n$-torus are isospectral deformations of spin structures on the commutative $n$-torus, we show that there is a big difference between their groups of symmetries. In the commutative case, a diffeomorphism acting from the torus can transform a spin structure into another according to the action of the diffeomorphism group as calculated in [15]. Here we show that in the noncommutative case when $n=2$ most spin structures, except the isospectral deformation of the trivial spin structure, are equivalent. When $n>2$ the result is less conclusive, due to insufficient knowledge of the full automorphism group of the $\mathrm{C}^{*}$-algebra in that case.

Recall the definition of a unitary equivalence between two spectral triples:

Definition (Unitary equivalence). A unitary equivalence between two spectral triples $(\mathcal{A}, H, D, J,(\Gamma))$ and $\left(\mathcal{A}, H, D^{\prime}, J^{\prime},\left(\Gamma^{\prime}\right)\right)$ is given by a unitary operator $W$ acting on the Hilbert space $H$ such that

$$
\begin{aligned}
W \pi(a) W^{-1} & =\pi(\sigma(a)) \text { for all } a \in \mathcal{A}, \\
W D W^{-1} & =D^{\prime}, \\
W J W^{-1} & =J^{\prime}, \\
W \Gamma W^{-1} & =\Gamma^{\prime},
\end{aligned}
$$

where $\sigma$ is a $*$-automorphism of the $\mathrm{C}^{*}$-algebra $A$ such that the algebra $\mathcal{A}$ is mapped into itself.

We first recall what is known about the automorphisms of the $\mathrm{C}^{*}$-algebra $A\left(\mathbb{T}_{\theta}^{n}\right)$. The main tool for understanding the automorphism group of a general noncommutative $n$-torus is [5], Theorem I, which tells us that for $\theta$ in a set which has full measure in the space of all antisymmetric matrices, the algebra $A\left(\mathbb{T}_{\theta}^{n}\right)$ is an inductive limit of direct sums of circle algebras. This results allows one to generalize a lot of results on noncommutative 2-tori to higher-dimensional tori. 
For a noncommutative $n$-torus which is an inductive limit of direct sums of circle algebras, the automorphism group fits in the following exact sequence [21], Theorem 2.1:

$$
1 \rightarrow \overline{\operatorname{Inn}}\left(A\left(\mathbb{\mathbb { T }}_{\theta}^{n}\right)\right) \rightarrow \operatorname{Aut}\left(A\left(\mathbb{T}_{\theta}^{n}\right)\right) \rightarrow \operatorname{Aut}\left(K\left(A\left(\mathbb{T}_{\theta}^{n}\right)\right)\right) \rightarrow 1 .
$$

Here an automorphism of $K_{0}\left(A\left(\mathbb{T}_{\theta}^{n}\right)\right) \oplus K_{1}\left(A\left(\mathbb{T}_{\theta}^{n}\right)\right)$ should preserve the order unit $\left[1_{A\left(\mathbb{T}_{\theta}^{n}\right)}\right]$ and the order structure $\left(K_{0}\left(A\left(\mathbb{T}_{\theta}^{n}\right)\right) \oplus K_{1}\left(A\left(\mathbb{T}_{\theta}^{n}\right)\right)\right)^{+}$.

The left side of the exact sequence can be further specified by [21], Corollary 4.6, which states that for algebras which are inductive limits of direct sums of circle algebras $\overline{\operatorname{Inn}}\left(A\left(\mathbb{T}_{\theta}^{n}\right)\right)=\overline{\operatorname{Inn}}_{0}\left(A\left(\mathbb{T}_{\theta}^{n}\right)\right)$. This means that an inner automorphism of a noncommutative torus can only give a unitary equivalence of two spin structures if the identity automorphism gives a unitary equivalence between the two spin structures.

By [30], Theorem 6.1, the order structure $\left(K_{0}\left(\mathcal{A}\left(\mathbb{T}_{\theta}^{n}\right)\right)\right)^{+}$consists precisely of those elements for which the normalized trace is positive, and by [18], Theorem 3.1, the image of this trace on $K_{0}$ is equal to the range of the exterior exponential of $\theta$ :

$$
\exp \bigwedge \theta=1 \oplus \theta \oplus \frac{1}{2}(\theta \wedge \theta) \oplus \cdots: \bigwedge^{\text {even }} \mathbb{Z}^{n} \rightarrow \mathbb{R} .
$$

For noncommutative 2-tori with $\theta$ irrational, this means that all automorphisms of $K\left(A\left(\mathbb{T}_{\theta}^{2}\right)\right)$ must be the identity on $K_{0}\left(A\left(\mathbb{\mathbb { T }}_{\theta}^{2}\right)\right)=\mathbb{Z}+\theta \mathbb{Z}$. The $K_{1}$-groups for noncommutative $n$-tori are also known to be isomorphic to $\mathbb{Z}^{2^{n-1}}$. For $n=2$ a partial lifting of $\operatorname{Aut}\left(\mathbb{Z}^{2}\right)=\mathrm{GL}(2, \mathbb{Z})$ is known [6], and given by the action of $\operatorname{SL}(2, \mathbb{Z})$ on the lattice $\mathbb{Z}^{2}$ of unitary generators $U_{\mathbf{x}}$. In [19] it is proven that in fact the whole automorphism group of the smooth algebra $\mathcal{A}\left(\mathbb{T}_{\theta}^{2}\right)$ for irrational $\theta$ with certain extra Diophantine conditions is given by a semidirect product of this action, the canonical torus action: $U_{e_{i}} \mapsto \lambda_{i} U_{e_{i}}$ and the projectivized group of unitaries of $\mathcal{A}\left(\mathbb{T}_{\theta}^{2}\right)$ in the connected component of the identity:

$$
\mathrm{PU}\left(\mathcal{A}\left(\mathbb{T}_{\theta}^{2}\right)\right)^{0} \rtimes\left(\mathbb{T}^{2} \rtimes \mathrm{SL}(2, \mathbb{Z})\right) .
$$

For $n>2$ the situation is less clear, since the action on the $K_{0}$-group need not be trivial anymore. When $n>2$, if $\theta$ is a matrix such that all $\theta_{i j}$ are independent over $\mathbb{Z}$, one can easily calculate that an outer automorphism cannot simply map basic unitaries to other basic unitaries. An automorphism $\sigma$ of this form must satisfy $e(\sigma(\mathbf{x}) \cdot \theta \sigma(\mathbf{y}))=e(\mathbf{x} \cdot \theta \mathbf{y})$ for all $\mathbf{x}, \mathbf{y} \in \mathbb{Z}^{n}$. If the $\theta_{i j}$ are independent over $\mathbb{Z}$, we see that $\sigma(\mathbf{x})=\alpha_{11} \mathbf{x}+\alpha_{12} \mathbf{y}$ and $\sigma(\mathbf{y})=\alpha_{21} \mathbf{x}+\alpha_{22} \mathbf{y}$ with $\left(\begin{array}{cc}\alpha_{11} & \alpha_{12} \\ \alpha_{21} & \alpha_{22}\end{array}\right) \in \operatorname{SL}(2, \mathbb{Z})$. When $n>2$ the only solution for all $\mathbf{x}$ and $\mathbf{y}$ is $\sigma(\mathbf{x})= \pm \mathbf{x}$ with sign the same for all $\mathbf{x}$.

With this knowledge of the automorphism group, we can proceed to our main theorem of this section.

Theorem B. Except for a set of $\theta$ of measure 0, the different spin structures of the smooth noncommutative $n$-torus $\mathcal{A}\left(\mathbb{T}_{\theta}^{n}\right)$ cannot be unitarily equivalent by an inner automorphism of the algebra. 
In the case $n=2$ the theorem was proven in [28], Theorem 2.5.

Proof. While for the proof of Theorem B it is only necessary to consider inner automorphisms, we get Corollary 2 if we also consider automorphisms induced by an action of $\operatorname{SL}(2, \mathbb{Z})$ on the algebra.

By [21], Corollary 4.6, and [5], Theorem I, we have that for almost all noncommutative tori $\overline{\operatorname{Inn}}\left(A\left(\mathbb{\nabla}_{\theta}^{n}\right)\right)=\overline{\operatorname{Inn}}_{0}\left(A\left(\mathbb{\nabla}_{\theta}^{n}\right)\right)$. This means that if an inner automorphism changes the spin structure, than so does the identity automorphism. We will assume in the following that the components of $\theta$ in the upper right corner are independent over $\mathbb{Z}$. The set of $\theta$ all of whose components in the upper right corner are independent over $\mathbb{Z}$ is of full measure, and so is the set of $\theta$ for which $\overline{\operatorname{Inn}}\left(\mathcal{A}\left(\mathbb{T}_{\theta}^{n}\right)\right)=\overline{\operatorname{Inn}}_{0}\left(\mathcal{A}\left(\mathbb{T}_{\theta}^{n}\right)\right)$, so their intersection also has full measure.

We label the basis of the Hilbert space for the different spin structures by the same labels $\mathbf{m}$, so $\boldsymbol{\mu}=\mathbf{m}+\boldsymbol{\epsilon}$. We see that for a spin structure $\boldsymbol{\epsilon}$ the operator $J$, written as in equation (17), acts by

$$
J_{\boldsymbol{\epsilon}} e_{\mathbf{m}, i}=\Lambda_{i j} e((\mathbf{m}+\boldsymbol{\epsilon}) \cdot \mathbf{A}(\mathbf{m}+\boldsymbol{\epsilon})) e_{-\mathbf{m}+2 \boldsymbol{\epsilon}, j}
$$

We consider a unitary transformation $W$ induced by an automorphism $\sigma \in$ $\operatorname{SL}(2, \mathbb{Z})$, we write

$$
W e_{\mathbf{0}, i}=\sum_{\mathbf{k}} \sum_{j} w_{\mathbf{k}, i j} e_{\mathbf{k}, j}
$$

for the action on the $e_{\mathbf{0}, i}$, and write $\sigma(\mathbf{x})$ for the obvious action of the automorphism $\sigma$ (either an element of $\operatorname{SL}(2, \mathbb{Z})$ when $n=2$, or the action $\mathbf{x} \mapsto \pm \mathbf{x}$ when $n>2$ ) on the vector $\mathbf{x} \in \mathbb{Z}^{n}$.

We first show that the action of the unitary transformation $W$ on the Hilbert space is fully determined by the action on the different $e_{0, i}$. Next we show that the condition (22c) implies that the action of the unitary transformation on the basis vectors must be such that a basis vector $e_{\mathbf{0}, i}$ is mapped to a linear combination of vectors $e_{\mathbf{k}, l}$ with $\mathbf{k}=\tilde{\boldsymbol{\epsilon}}-\sigma(\boldsymbol{\epsilon})$, where $\boldsymbol{\epsilon}$ is the original spin structure and $\tilde{\boldsymbol{\epsilon}}$ the new spin structure. If $\sigma(\boldsymbol{\epsilon})= \pm \boldsymbol{\epsilon}$, this means that the spin structure is unchanged, since $\mathbf{k}$ must lie in $\mathbb{Z}^{n}$. For the noncommutative 2-torus, we have $\sigma \in \operatorname{SL}(2, \mathbb{Z})$, and we see that if $\epsilon \in \mathbb{Z}^{2}$, the spin structure is unchanged. All spin structures on the noncommutative 2-torus for which $\epsilon \notin \mathbb{Z}^{2}$ are unitarily equivalent.

Consider a unitary transformation $W$ that maps a spin structure $\boldsymbol{\epsilon}$ to $\tilde{\boldsymbol{\epsilon}}$. Since $e_{\mathbf{x}, i}=U_{\mathbf{x}} e(-\mathbf{x} \cdot \mathbf{A x} / 2-\mathbf{x} \cdot \mathbf{A} \boldsymbol{\epsilon}) e_{\mathbf{0}, i}$ and using (22a) we can write

$$
\begin{aligned}
W e_{\mathbf{m}, i}= & W U_{\mathbf{m}} e(-\mathbf{m} \cdot \mathbf{A m} / 2-\mathbf{m} \cdot \mathbf{A} \boldsymbol{\epsilon}) e_{\mathbf{0}, i} \\
= & U_{\sigma(\mathbf{m})} e(-\mathbf{m} \cdot \mathbf{A m} / 2-\mathbf{m} \cdot \mathbf{A} \boldsymbol{\epsilon})\left(\sum_{\mathbf{k}, j} w_{\mathbf{k}, i j} e_{\mathbf{k}, j}\right) \\
= & \sum_{\mathbf{k}, j} e\left(\sigma(\mathbf{m}) \cdot \mathbf{A}(\mathbf{k}+\tilde{\boldsymbol{\epsilon}})+\frac{1}{2} \sigma(\mathbf{m}) \cdot \mathbf{A} \sigma(\mathbf{m})\right. \\
& -\mathbf{m} \cdot \mathbf{A m} / 2-\mathbf{m} \cdot \mathbf{A} \boldsymbol{\epsilon}) w_{\mathbf{k}, i j} e_{\mathbf{k}+\sigma(\mathbf{m}), j} .
\end{aligned}
$$


So the action of $W$ on the Hilbert space is fully determined by the action on the $e_{\mathbf{0}, i}$. Requirement (22c) gives the following equations:

$$
\begin{aligned}
& W J_{\boldsymbol{\epsilon}} e_{\mathbf{m}, i}= \Lambda_{i j} e((\mathbf{m}+\boldsymbol{\epsilon}) \cdot \mathbf{A}(\mathbf{m}+\boldsymbol{\epsilon})) W e_{-\mathbf{m}+2 \boldsymbol{\epsilon}, j} \\
&= \sum_{\mathbf{k}, l} \Lambda_{i j} e\left((\mathbf{m}+\boldsymbol{\epsilon}) \cdot \mathbf{A}(\mathbf{m}+\boldsymbol{\epsilon})+\sigma(-\mathbf{m}+2 \boldsymbol{\epsilon}) \cdot \mathbf{A}\left(\mathbf{k}+\tilde{\boldsymbol{\epsilon}}+\frac{1}{2} \sigma(-\mathbf{m}\right.\right. \\
&+2 \boldsymbol{\epsilon}))) \cdot e\left(-(2 \boldsymbol{\epsilon}-\mathbf{m}) \cdot \mathbf{A}\left(\boldsymbol{\epsilon}+\frac{1}{2}(2 \boldsymbol{\epsilon}-\mathbf{m})\right)\right) w_{\mathbf{k}, j l} e_{\mathbf{k}+\sigma(-\mathbf{m}+2 \boldsymbol{\epsilon}), l} \\
&= \sum_{\mathbf{k}, l} \Lambda_{i j} e(\boldsymbol{\epsilon} \cdot \mathbf{A}(-3 \boldsymbol{\epsilon}+2 \mathbf{m})+\sigma(\boldsymbol{\epsilon}) \cdot \mathbf{A}(2(\tilde{\boldsymbol{\epsilon}}+\mathbf{k})+\sigma(2 \boldsymbol{\epsilon}-\mathbf{m}))) \\
& \cdot e\left(\mathbf{m} \cdot \mathbf{A}\left(3 \boldsymbol{\epsilon}+\frac{1}{2} \mathbf{m}\right)+\sigma(\mathbf{m}) \cdot \mathbf{A}(-\tilde{\boldsymbol{\epsilon}}-\mathbf{k}\right. \\
&\left.\left.+\sigma\left(\frac{1}{2} \mathbf{m}-\boldsymbol{\epsilon}\right)\right)\right) w_{\mathbf{k}, j l} e_{\mathbf{k}+\sigma(-\mathbf{m}+2 \boldsymbol{\epsilon}), l,} \\
& J_{\tilde{\boldsymbol{\epsilon}} W e_{\mathbf{m}, i}=} \sum_{\mathbf{k}, j} e(\sigma(\mathbf{m}) \cdot \mathbf{A}(\mathbf{k}+\tilde{\boldsymbol{\epsilon}}+\sigma(\mathbf{m}) / 2)-\mathbf{m} \cdot \mathbf{A}(\boldsymbol{\epsilon}+\mathbf{m} / 2)) w_{\mathbf{k}, i j} e_{\mathbf{k}+\sigma(\mathbf{m}), j} \\
&=\sum_{\mathbf{k}, j} \Lambda_{j l} e((\mathbf{k}+\sigma(\mathbf{m})+\tilde{\boldsymbol{\epsilon}}) \cdot \mathbf{A}(\mathbf{k}+\sigma(\mathbf{m})+\tilde{\boldsymbol{\epsilon}})) \cdot e(-\sigma(\mathbf{m}) \\
&\cdot \mathbf{A}(\mathbf{k}+\tilde{\boldsymbol{\epsilon}}+\sigma(\mathbf{m}) / 2)+\mathbf{m} \cdot \mathbf{A}(\boldsymbol{\epsilon}+\mathbf{m} / 2)) w_{\mathbf{k}, i j}^{*} e_{-\mathbf{k}-\sigma(\mathbf{m})+2 \tilde{\boldsymbol{\epsilon}}, j} \\
&= \sum_{\mathbf{k}, j} \Lambda_{j l} e((\tilde{\boldsymbol{\epsilon}}+\mathbf{k}) \cdot \mathbf{A}(\tilde{\boldsymbol{\epsilon}}+\mathbf{k}+\sigma(\mathbf{m}))+\mathbf{m} \cdot \mathbf{A}(\boldsymbol{\epsilon}+\mathbf{m} / 2)) \\
& \cdot e\left(\frac{1}{2} \sigma(\mathbf{m}) \cdot \mathbf{A} \sigma(\mathbf{m})\right) w_{\mathbf{k}, i j}^{*} e_{-\mathbf{k}-\sigma(\mathbf{m})+2 \tilde{\boldsymbol{\epsilon}}, j} .
\end{aligned}
$$

Collecting the vectors with the same indices and in the same Hilbert space $\mathscr{H}^{j}$ we see that for indices $\mathbf{k}+\sigma(-\mathbf{m}+2 \boldsymbol{\epsilon})=-\mathbf{k}^{\prime}-\sigma(\mathbf{m})+2 \tilde{\boldsymbol{\epsilon}}$, or $\mathbf{k}^{\prime}=-\mathbf{k}+2 \tilde{\boldsymbol{\epsilon}}-2 \sigma(\boldsymbol{\epsilon})$ :

$$
\begin{aligned}
& e\left(\sigma(-\mathbf{m}+2 \boldsymbol{\epsilon}) \cdot \mathbf{A}\left(\mathbf{k}+\tilde{\boldsymbol{\epsilon}}+\frac{1}{2} \sigma(-\mathbf{m}+2 \boldsymbol{\epsilon})\right)-(2 \boldsymbol{\epsilon}-\mathbf{m}) \cdot \mathbf{A}\left(\boldsymbol{\epsilon}+\frac{1}{2}(2 \boldsymbol{\epsilon}-\mathbf{m})\right)\right) w_{\mathbf{k}, j l} l \\
& =\Lambda_{j l} e\left((\tilde{\boldsymbol{\epsilon}}+\mathbf{k}) \cdot \mathbf{A}(\tilde{\boldsymbol{\epsilon}}+\mathbf{k}+\sigma(\mathbf{m}))+\mathbf{m} \cdot \mathbf{A}(\boldsymbol{\epsilon}+\mathbf{m} / 2)+\frac{1}{2} \sigma(\mathbf{m}) \cdot \mathbf{A} \sigma(\mathbf{m})\right) w_{\mathbf{k}, i j}^{*}
\end{aligned}
$$

Since $\Lambda^{-1}=\Lambda^{\dagger}$ and $\sum_{j} \Lambda_{l j} \Lambda_{j i}^{*}= \pm \delta_{i l}$ with $\delta_{i l}$ the Kronecker delta, this implies that

$$
\begin{aligned}
& w_{\mathbf{k}, i j} \\
& = \pm e(2 \boldsymbol{\epsilon} \cdot \mathbf{A}(3 \boldsymbol{\epsilon}-2 \mathbf{m})+3 \tilde{\boldsymbol{\epsilon}} \cdot \mathbf{A}(3 \tilde{\boldsymbol{\epsilon}}-\mathbf{k}+\sigma(-2 \boldsymbol{\epsilon}+\mathbf{m}))) \\
& \cdot e(\mathbf{k} \cdot \mathbf{A}(-3 \tilde{\boldsymbol{\epsilon}}+\mathbf{k}+\sigma(2 \boldsymbol{\epsilon}-\mathbf{m}))+\sigma(\boldsymbol{\epsilon}) \cdot \mathbf{A}(-4 \tilde{\boldsymbol{\epsilon}}+4 \mathbf{k}+3 \sigma(2 \boldsymbol{\epsilon}-\mathbf{m}))) \\
& \quad \cdot e(\sigma(\mathbf{m}) \cdot \mathbf{A}(\tilde{\boldsymbol{\epsilon}}+\mathbf{k}+\sigma(\boldsymbol{\epsilon}))-2 \mathbf{m} \cdot \mathbf{A} \boldsymbol{\epsilon}) w_{-\mathbf{k}+2 \tilde{\boldsymbol{\epsilon}}-2 \sigma(\boldsymbol{\epsilon}), i j}^{*} .
\end{aligned}
$$

Applying this same formula again for $w_{-k+2 \tilde{\boldsymbol{\epsilon}}-2 \sigma(\boldsymbol{\epsilon}), i j}^{*}$, we get

$$
\begin{aligned}
& w_{-\mathbf{k}+2 \tilde{\boldsymbol{\epsilon}}-2 \sigma(\boldsymbol{\epsilon}), i j}^{*} \\
& =e(\boldsymbol{\epsilon} \cdot \mathbf{A}(3 \boldsymbol{\epsilon}-2 \mathbf{m})-(\tilde{\boldsymbol{\epsilon}}+\mathbf{k}) \cdot \mathbf{A}(\tilde{\boldsymbol{\epsilon}}+\mathbf{k}+\sigma(\mathbf{m}))-\mathbf{m} \cdot \mathbf{A}(4 \boldsymbol{\epsilon}+\mathbf{m})) \\
& \quad \cdot e(\sigma(\boldsymbol{\epsilon}) \cdot \mathbf{A}(6 \tilde{\boldsymbol{\epsilon}}-2 \mathbf{k}-\sigma(2 \boldsymbol{\epsilon}-\mathbf{m}))+\sigma(\mathbf{m}) \cdot \mathbf{A}(3 \tilde{\boldsymbol{\epsilon}}-\mathbf{k}-\sigma(\boldsymbol{\epsilon}+\mathbf{m}))) w_{\mathbf{k}, j k} .
\end{aligned}
$$


Filling in the expression of (24) in (23), we see

$$
\begin{aligned}
w_{\mathbf{k}, j k}= & e(2 \tilde{\boldsymbol{\epsilon}} \cdot \mathbf{A}(4 \tilde{\boldsymbol{\epsilon}}-2 \mathbf{k}+\sigma(\mathbf{m}-3 \boldsymbol{\epsilon}))+2 \mathbf{k} \cdot \mathbf{A}(\sigma(\boldsymbol{\epsilon}-\mathbf{m})-2 \tilde{\boldsymbol{\epsilon}})) \\
& \cdot e(2 \sigma(\boldsymbol{\epsilon}) \cdot \mathbf{A}(3 \mathbf{k}-5 \tilde{\boldsymbol{\epsilon}}+\sigma(4 \boldsymbol{\epsilon}-\mathbf{m}))+2 \sigma(\mathbf{m}) \cdot \mathbf{A}(\mathbf{k}-\tilde{\boldsymbol{\epsilon}}+\sigma(\boldsymbol{\epsilon}))) w_{\mathbf{k}, j k} .
\end{aligned}
$$

Collecting all terms which contain $\mathbf{m}$ we observe that these add up to $2 \sigma(\mathbf{m}) \cdot \theta(\mathbf{k}+$ $\sigma(\boldsymbol{\epsilon})-\tilde{\boldsymbol{\epsilon}})$. Since the equality above should hold for all $\mathbf{m}$, this means that either $w_{\mathbf{k}, i j}=0$, or $\mathbf{m} \cdot \theta(\mathbf{k}+\sigma(\boldsymbol{\epsilon})-\tilde{\boldsymbol{\epsilon}})=0$ for all $\mathbf{m}$.

If all components of $\theta$ in the upper right corner are independent over $\mathbb{Z}$ this can only be the case if $\mathbf{k}+\sigma(\boldsymbol{\epsilon})-\tilde{\boldsymbol{\epsilon}}=0$, hence $\mathbf{k}=\tilde{\boldsymbol{\epsilon}}-\sigma(\boldsymbol{\epsilon})$. Since $\mathbf{k}$ must lie in $\mathbb{Z}^{n}$, we see that if $\sigma(\boldsymbol{\epsilon})= \pm \boldsymbol{\epsilon}$ the spin structure cannot change.

From the proof it also follows:

Corollary 2. Let $\sigma \in \operatorname{SL}(2, \mathbb{Z})$. The automorphism $\sigma$ of $\mathbb{Z}^{2}$ induces an automorphism of the noncommutative 2-torus of the form $U_{\mathbf{x}} \mapsto U_{\sigma(\mathbf{x})}$.

This automorphism induces a unitary equivalence of real spectral triples which maps a spin structure $\boldsymbol{\epsilon}$ to $\tilde{\boldsymbol{\epsilon}}=\sigma(\boldsymbol{\epsilon})$. In particular, real spectral triples on the noncommutative 2-torus which are not isospectral deformations of the trivial spin structure on the commutative 2-torus are unitary equivalent to each other, via the unitary map $W$, defined by

$$
\begin{aligned}
W e_{\boldsymbol{\mu}} & =e_{\sigma(\boldsymbol{\mu})}, \\
W U_{\mathbf{x}} W^{-1} & =U_{\sigma(\mathbf{x})}, \\
D^{\prime} & =\left(\sum_{j} \sigma^{-1}\left(\boldsymbol{\tau}_{j}\right) \cdot \boldsymbol{\delta}\right) \otimes A_{j}, \\
J^{\prime} & =J
\end{aligned}
$$

composed with an additional unitary map given by Lemma 1 that maps the representation $\pi^{\mathbf{A}^{\prime}}$ with $\mathbf{A}^{\prime}=\sigma^{t} \mathbf{A} \sigma$ to the original $\pi^{\mathbf{A}}$.

Proof. The statement about the spin structures follows from the statements at the end of the proof of Theorem B. Since $\sigma \in \operatorname{SL}(2, \mathbb{Z})$ is an automorphism of the lattice $\mathbb{Z}^{2}$, it cannot map an $\epsilon \notin \mathbb{Z}^{2}$ to one in $\mathbb{Z}^{2}$ and vice-versa. On the other hand, set $\boldsymbol{\epsilon}_{10}=\left(\begin{array}{c}1 / 2 \\ 0\end{array}\right), \boldsymbol{\epsilon}_{01}=\left(\begin{array}{c}0 \\ 1 / 2\end{array}\right), \boldsymbol{\epsilon}_{11}=\left(\begin{array}{l}1 / 2 \\ 1 / 2\end{array}\right)$, and $M=\left(\begin{array}{rr}1 & 0 \\ -1 & 1\end{array}\right), N=\left(\begin{array}{cc}1 & -1 \\ 0 & 1\end{array}\right)$ in $\operatorname{SL}(2, \mathbb{Z})$. We see

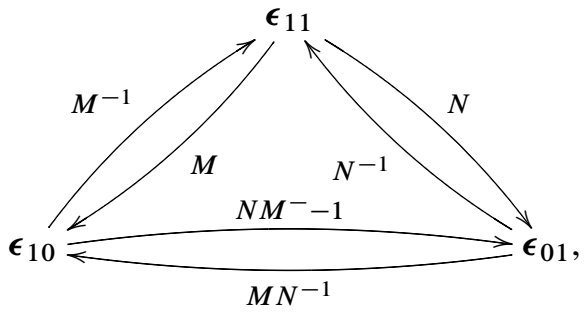


where the matrices $M$ and $N$ act by left multiplication, so there exist $\sigma \in \operatorname{SL}(2, \mathbb{Z})$ such that the $\epsilon \notin \mathbb{Z}^{2}$ are mapped to one another. Given a unitary operator $W e_{\mu}=$ $e_{\sigma(\mu)}$, we can calculate its action on the Dirac operator by using the definition (22b):

$$
\begin{aligned}
D^{\prime} e_{\boldsymbol{\mu}, k} & =W D W^{-1} e_{\boldsymbol{\mu}, k} \\
& =W D e_{\sigma^{-1}(\boldsymbol{\mu}), k} \\
& =W \sum_{j}\left(\boldsymbol{\tau}_{j} \cdot\left(\sigma^{-1}(\boldsymbol{\mu})\right)\right) \otimes A_{j} e_{\sigma^{-1}}(\boldsymbol{\mu}), k \\
& =\sum_{j}\left(\sigma^{-1}\left(\boldsymbol{\tau}_{j}\right) \cdot \boldsymbol{\mu}\right) \otimes A_{j} e_{\boldsymbol{\mu}, k} .
\end{aligned}
$$

The action of the automorphisms $\sigma \in \operatorname{SL}(2, \mathbb{Z})$ on the Dirac operator when $\epsilon=0$ was also determined for $\epsilon=0$ in [37], Section 7.1. with the change in notation that our $\boldsymbol{\tau}_{1}$ is given there by $\left(\begin{array}{c}0 \\ \operatorname{Im} \tau\end{array}\right)$ and our $\boldsymbol{\tau}_{2}$ is there $\left(\begin{array}{c}1 \\ \operatorname{Re} \tau\end{array}\right)$.

Some questions left unanswered by these results are the effects of the outer automorphisms for $n>2$ and Morita equivalences of noncommutative tori, as described in [32] and [20], on the spin structure. Also, the definition of an equivariant spectral triple can be generalized to allow $[D, h] \neq 0$, but bounded. This was used to construct equivariant $U_{q}(\mathrm{SU}(2))$ spectral triples in [14]. It would be interesting to investigate what possibilities this would open up for the noncommutative torus. We hope to return to these questions in the future.

\section{References}

[1] J. F. Adams, Vector fields on spheres. Ann. of Math. (2) 75 (1962), 603-632. Zbl 0112.38102 MR 0139178

[2] J. F. Adams, P. D. Lax, and R. S. Phillips, On matrices whose real linear combinations are non-singular. Proc. Amer. Math. Soc. 16 (1965), 318-322. Zbl 0168.02404 MR 0179183

[3] H. Aoki, J. Nishimura, and Y. Susaki, The index of the overlap Dirac operator on a discretized 2d non-commutative torus. J. High Energy Phys. (2007), 033. MR 2318006

[4] J. Bellissard, A. van Elst, and H. Schulz-Baldes, The noncommutative geometry of the quantum Hall effect. J. Math. Phys. 35 (1994), 5373-5451. Zbl 0824.46086 MR 1295473

[5] F. P. Boca, The structure of higher-dimensional noncommutative tori and metric Diophantine approximation. J. Reine Angew. Math. 492 (1997), 179-219. Zbl 0884.46040 MR 1488068

[6] B. A. Brenken, Representations and automorphisms of the irrational rotation algebra. Pacific J. Math. 111 (1984), 257-282. Zbl 0576.46051 MR 734854

[7] A. Connes, Noncommutative geometry. Academic Press, San Diego, CA, 1994. Zbl 0818.46076 MR 1303779

[8] A. Connes, Noncommutative geometry and reality. J. Math. Phys. 36 (1995), 6194-6231. Zbl 0871.58008 MR 1355905 
[9] A. Connes, Gravity coupled with matter and the foundation of non-commutative geometry. Comm. Math. Phys. 182 (1996), 155-176. Zbl 0881.58009 MR 1441908

[10] A. Connes, On the spectral characterization of manifolds. J. Noncommut. Geom. 7 (2013), 1-82. Zbl 6155603 MR 3032810

[11] A. Connes and M. Dubois-Violette, Noncommutative finite-dimensional manifolds. I. Spherical manifolds and related examples. Comm. Math. Phys. 230 (2002), 539-579. Zbl 1026.58005 MR 1937657

[12] A. Connes and M. Marcolli, Noncommutative geometry, quantum fields and motives. Amer. Math. Soc. Colloq. Publ. 55, Amer. Math. Soc., Providence, RI, 2008. Zbl 1209.58007 MR 2371808

[13] L. Dạbrowski, Spinors and theta deformations, Russ. J. Math. Phys. 16 (2009), no. 3, 404-408.

[14] L. Dạbrowski, F. D’A Andrea, G. Landi, and E. Wagner, Dirac operators on all Podleś quantum spheres. J. Noncommut. Geom. 1 (2007), 213-239. Zbl 1121.58008 MR 2308305

[15] L. Dạbrowski and R. Percacci, Spinors and diffeomorphisms. Comm. Math. Phys. 106 (1986), 691-704. Zbl 0605.53042 MR 860317

[16] S. Disney, G. A. Elliott, A. Kumjian, and I. Raeburn, On the classification of noncommutative tori. C. R. Math. Rep. Acad. Sci. Canada 7 (1985), 137-141. Zbl 0573.46036 MR 781813

[17] E. G. Effros and F. Hahn, Locally compact transformation groups and $C^{*}$ - algebras. Mem. Amer. Math. Soc. 75, Amer. Math. Soc., Providence, R.I., 1967. Zbl 0184.17002 MR 0227310

[18] G. A. Elliott, On the $K$-theory of the $C^{*}$-algebra generated by a projective representation of a torsion-free discrete abelian group. In Operator algebras and group representations, Vol. I (Neptun, 1980), Monogr. Stud. Math. 17, Pitman, Boston 1984, 157-184. Zbl 0542.46030 MR 731772

[19] G. A. Elliott, The diffeomorphism group of the irrational rotation $C^{*}$-algebra. C. R. Math. Rep. Acad. Sci. Canada 8 (1986), 329-334. Zbl 0617.46068 MR 859436

[20] G. A. Elliott and H. Li, Morita equivalence of smooth noncommutative tori. Acta Math. 199 (2007), 1-27. Zbl 1137.46030 MR 2350069

[21] G. A. Elliott and M. Rørdam, The automorphism group of the irrational rotation $C^{*}$ algebra. Comm. Math. Phys. 155 (1993), 3-26. Zbl 0895.46029 MR 1228523

[22] J. M. Gracia-Bondía, J. C. Várilly, and H. Figueroa, Elements of noncommutative geometry. Birkhäuser Adv. Texts, Birkhäuser, Boston 2001. Zbl 0958.46039 MR 1789831

[23] W.-c. Hsiang and C. T. C. Wall, On homotopy tori. II. Bull. London Math. Soc. 1 (1969), 341-342. Zbl 0176.22003 MR 0258044

[24] C. Kassel, Quantum groups. Graduate Texts in Math. 155, Springer-Verlag, New York 1995. Zbl 0808.17003 MR 1321145

[25] G. Landi, F. Lizzi, and R. J. Szabo, String geometry and the noncommutative torus. Comm. Math. Phys. 206 (1999), 603-637. Zbl 0949.46037 MR 1721895

[26] H. B. Lawson, Jr. and M.-L. Michelsohn, Spin geometry. Princeton Math. Ser. 38, Princeton University Press, Princeton, NJ, 1989. Zbl 0688.57001 MR 1031992 
[27] J. Milnor, Spin structures on manifolds. Enseignement Math. (2) 9 (1963), 198-203. Zbl 0116.40403 MR 0157388

[28] M. Paschke and A. Sitarz, On Spin structures and Dirac operators on the noncommutative torus. Lett. Math. Phys. 77 (2006), 317-327. Zbl 1120.58008 MR 2260377

[29] M. Reed and B. Simon, Methods of modern mathematical physics IV: Analysis of operators. Academic Press, New York 1978. Zbl 0401.47001 MR 0493421

[30] M. A. Rieffel, Projective modules over higher-dimensional noncommutative tori. Canad. J. Math. 40 (1988), 257-338. Zbl 0663.46073 MR 941652

[31] M. A. Rieffel, Non-commutative tori - a case study of non-commutative differentiable manifolds. In Geometric and topological invariants of elliptic operators (Brunswick, ME, 1988), Contemp. Math. 105, Amer. Math. Soc., Providence, RI, 1990, 191-211. Zbl 0713.46046 MR 1047281

[32] M. A. Rieffel and A. Schwarz, Morita equivalence of multidimensional noncommutative tori. Internat. J. Math. 10 (1999), 289-299. Zbl 0968.46060 MR 1687145

[33] W. M. Schmidt, Diophantine approximations and Diophantine equations. Lecture Notes in Math. 1467, Springer-Verlag, Berlin 1991. Zbl 0754.11020 MR 1176315

[34] A. Sitarz, Equivariant spectral triples. In Noncommutative geometry and quantum groups (Warsaw, 2001), Banach Center Publ. 61, Polish Acad. Sci., Warsaw 2003, 231-263. Zbl 1061.58007 MR 2024433

[35] M. Takesaki, Tomita's theory of modular Hilbert algebras and its applications. Lecture Notes in Math. 128, Springer-Verlag, Berlin 1970. Zbl 0193.42502 MR 0270168

[36] M. Takesaki, Theory of operator algebras. I. Springer-Verlag, New York 1979. Zbl 0436.46043 MR 548728

[37] J. C. Várilly, An introduction to noncommutative geometry. EMS Ser. Lect. Math., European Math. Soc. (EMS), Zürich 2006. Zbl 1097.58004 MR 2239597 (2007e:58011)

[38] M. Wambst, Hochschild and cyclic homology of the quantum multiparametric torus. $J$. Pure Appl. Algebra 114 (1997), 321-329. Zbl 0881.18014 MR 1426492

Received April 19, 2011; revised July 25, 2011

J. J. Venselaar, Mathematics 253-37, Caltech, Pasadena, CA 91125, U.S.A.

E-mail: janjitse@caltech.edu 